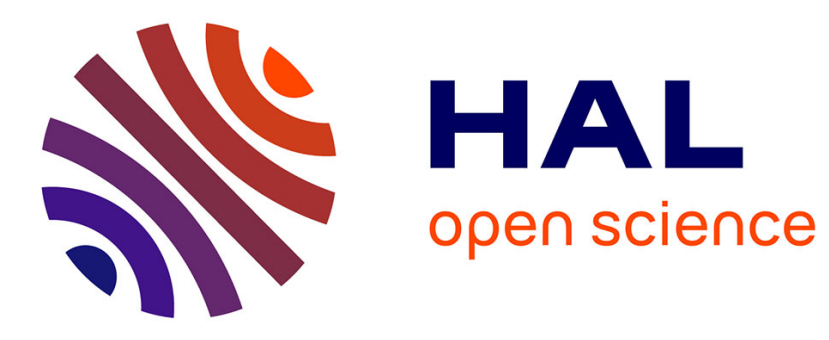

\title{
Visual Servoing of Robot Manipulators, Part I: Projective Kinematics
}

\author{
Andreas Ruf, Radu Horaud
}

\section{To cite this version:}

Andreas Ruf, Radu Horaud. Visual Servoing of Robot Manipulators, Part I: Projective Kinematics. The International Journal of Robotics Research, 1999, 18 (11), pp.1101-1118. 10.1177/02783649922067744 . inria-00590106

\section{HAL Id: inria-00590106 https://hal.inria.fr/inria-00590106}

Submitted on 3 May 2011

HAL is a multi-disciplinary open access archive for the deposit and dissemination of scientific research documents, whether they are published or not. The documents may come from teaching and research institutions in France or abroad, or from public or private research centers.
L'archive ouverte pluridisciplinaire HAL, est destinée au dépôt et à la diffusion de documents scientifiques de niveau recherche, publiés ou non, émanant des établissements d'enseignement et de recherche français ou étrangers, des laboratoires publics ou privés. 


\title{
Visual Servoing of Robot Manipulators Part I: Projective Kinematics
}

\author{
Andreas Ruf* \\ Radu Horaud* \\ Andreas.Ruf@inrialpes.fr $\quad$ Radu.Horaud@inrialpes.fr \\ GRAVIR-IMAG, INRIA Rhone-Alpes \\ 655, avenue de l'Europe \\ 38330 Montbonnot St.Martin, France
}

December 5, 2000

\begin{abstract}
Visual servoing of robot manipulators is a key technique where the appearance of an object in the image plane is used to control the velocity of the endeffector such that the desired position is reached in the scene. The vast majority of visual servoing methods proposed so far uses calibrated robots in conjunction with calibrated cameras. It has been shown that the behavior of visual control loops does not degrade too much in the presence of calibration errors. Nevertheless, camera and robot calibration are complex and time-consuming processes requiring special-purpose mechanical devices, such as theodolites and calibration jigs.
\end{abstract}

In this paper we suggest to formulate a visual servoing control loop in a non-metric space, which in our case amounts to the projective space in which a triangulation of the scene using an uncalibrated stereo rig is expressed. The major consequence of controlling the robot in non-metric space rather than in Euclidean space, is that both the robot's direct kinematic map and the robot's Jacobian matrix must be defined in this space as well.

The elementary joint-space motions that can be performed by a robot manipulator are pure rotations and pure translations. Traditionally, these

${ }^{*}$ This work is supported by the European Esprit programme through the VIGOR project Esprit-IV LTR number 26247. Andreas Ruf is supported by the Marie-Curie (TMR) Fellowship FMBICT972281 motions are represented as Euclidean transformations. Since these motions are observed with an uncalibrated stereo rig, it will be convenient to represent them as projective transformations (homographies) rather than Euclidean transformations. Indeed, it will be shown that rotations and translations can be parameterized as special cases of homographies which will be called projective rotations and projective translations. The algebraic properties of this non-metric representation of elementary motions will be thoroughly investigated allowing to characterize the direct kinematic map and the Jacobian matrix of a manipulator. Therefore we introduce the concepts of projective kinematics and a projective Jacobian matrix. Unlike the classical robot Jacobian matrix of a manipulator which relates the robot joint-velocities to the kinematic screw associated with the end-effector, we establish a direct relationship between joint-velocities and image-plane velocities. The latter are velocities associated with image points arising from the 3-D to 2-D projection of end-effector points.

Finally, we provide a practical method to estimate the projective kinematic model and we describe some preliminary simulated experiments which use this non-metric model to perform stereobased servoing. Nevertheless, in depth analysis of projective control will be the topic of a forthcoming paper.

keywords: projective geometry, uncalibrated vision, kinematics, stereo vision, articulated motion, visual servoing 


\section{Introduction}

Advanced automation requires intelligent robots that are able to manipulate, grasp, inspect, weld, and machine objects in unstructured environments. In the past, a variety of sensors have been used for the purpose of real-time robot control, including force/torque tactile sensors, laser triangulation devices, proximity (lateral-effect Photo-diode), sonar (both Polaroid and FM), and infrared phase or time-of-flight range sensors. For various reasons none of these sensors are wholly satisfactory and all have major weaknesses. Computer vision can provide powerful geometric cues to help guide and position robots and their tools. With respect to the sensors just cited, vision has several advantages: (i) it has high angular resolution; (ii) it is increasingly inexpensive, has high reliability, and the sensors are of low weight with low power consumption; (iii) it is passive, with a large range of possible light wavelengths; (iv) it can operate over a substantial range, from 0.5 meters to tens of meters (with the same lens); and (v) the bandwidth of a vision system is compatible with the robot controllers at the task level.

A key technique is visual servoing where the appearance of a target in the image is used to control the position of the end-effector and to move it to a desired position in the scene [17]. More generally, visual servoing is an appealing technique which, with the increased speed of processing available today, enables the loop to be closed between sensing and action so that a robot's behavior can be modified on-line according to what it sees [26].

The vast majority of visual servoing methods proposed so far uses calibrated robots in conjunction with calibrated cameras. Nevertheless, camera and robot calibration are complex and timeconsuming processes requiring special-purpose mechanical devices, such as theodolites and calibration rigs [22], [28, 14, 4]. Therefore, from a practical point of view, it is convenient to be able to cope with a camera-robot setup for which accurate calibration data are not necessarily available. A technique which tolerates such calibration errors is image-based servoing and a number of approaches were suggested in the past using either hand-held cameras [5] or cameras mounted onto independent fixtures $[11,7,15]$.
The approach envisaged in this paper uses an uncalibrated stereo rig (a pair of cameras that are rigidly attached to each other but for which neither intrinsic nor extrinsic parameters are computed in advance) which is either mounted onto a robot or mounted onto an independent fixture. When such a stereo rig observes an unknown $3-\mathrm{D}$ scene, it is possible to compute its epipolar geometry [20] and to perform 3-D reconstruction in projective space [9].

A number of authors have suggested to recover camera parameters and Euclidean structure by upgrading this projective reconstruction to metric reconstruction. The projective-to-metric upgrade can be done with either a single camera [21], [10], [23] or a stereo camera pair [29], [3], [12], [13].

Rather than upgrading this projective representation to a metric one we propose to perform the visual servo control loop in non-metric space - a special subspace of the projective space which will be investigated. The major consequence of controlling the robot in non-metric space is that the robot's direct kinematic map as well as the the robot's Jacobian matrix must be described in this space as well.

The elementary joint-space motions that can be performed by a robot manipulator are pure rotations and pure translations. Traditionally, these motions are represented as Euclidean transformations. Since these motions are observed with an uncalibrated stereo rig, it will be convenient to represent them as projective transformations (homographies) rather than Euclidean transformations. Indeed, it will be shown that rotations and translations can be parameterized as special cases of homographies which will be called projective rotations and projective translations. The algebraic properties of this non-metric representation of elementary motions will allow us to characterize the direct kinematic map and the Jacobian matrix of a manipulator. Unlike the classical Jacobian matrix of a manipulator which relates robot-joint velocities to the kinematic screw associated with the endeffector, we establish a relationship between joint velocities and image-plane velocities. The latter are velocities associated with image points arising from the 3-D to 2-D projection of end-effector points.

The direct kinematic map together with the 
motor-to-image Jacobian matrix to be defined lateron will be referred to as the projective kinematics of a robot manipulator. They are the basis of the design of projective control which consists of visually servoing a robot with a pair of cameras and which will be the topic of a forthcoming article.

To date, there are very few attempts to design non-metric visual control loops. In [19] an uncalibrated stereo rig is fixed onto a robot arm and camera self-calibration is performed prior to the execution of the control loop. In [18] a visual-motor Jacobian model is estimated and refined on-line the setup involves a stereo rig observing a multifingered hand mounted onto a robot arm. Since such an empirical model is only locally valid, a collection of Jacobians represents a piecewise linear model of the part of the visual-motor space actually explored. Such an approach leads to non-linear optimization and does not guarantee that the robot's kinematics and its associated Jacobian are correctly modeled.

\subsection{Paper contribution and organi- zation}

The reminder of the paper is organized as follows. Section 2 reminds some properties of algebraic similarity and of the real Jordan decomposition of various types of rigid displacements. Section 3 reminds the algebraic geometry of a stereo camera pair with known epipolar geometry and the principle of projective reconstruction.

Section 4 introduces the concept of projective displacement, i.e., the projective transformations conjugate to rigid motions. Important properties of projective displacements such as their normalization, their Lie group structure, and their associated tangent operators are defined and analyzed. Moreover, we analyze the action of projective displacements onto the $3-\mathrm{D}$ projective space which allows us to define the non-metric velocity of a point in projective space. The special cases of projective translations and projective rotations are thoroughly studied in sections 5 and 6 .

Section 7 introduces the projective geometry of a stereo-robot configuration which allows us to define the concept of projective kinematics associated with an articulated mechanism such as a robot whose articulations are either prismatic or revolute joints. To complete the non-metric kinematic model, the projective Jacobian matrix of an articulated mechanism is defined and described in detail in section8. Section 9 suggests a numerical method to estimate in practice the projective kinematic map, which amounts to a "projective calibration" of a robot. Section 10 presents a simulated example of visually servoing a robot using the projective modeling (projective kinematics and projective Jacobian) introduced in sections 8 and 9 . Finally, section 11 gives some directions for future work.

\section{Preliminaries}

The following sections recapitulate the principle of the similarity of matrix transforms. They are made explicit for matrix representations of rigid displacement and corresponding homographies of projective space. The later are introduced in the section 3 on three-dimensional reconstruction from uncalibrated stereo vision.

\subsection{Algebraic similarity}

Two square matrices $\mathbf{A}$ and $\mathbf{B}$ are conjugate or similar, if a non-singular matrix $\mathbf{X}$ exists, such that

$$
\mathbf{B}=\mathbf{X}^{-1} \mathbf{A} \mathbf{X}
$$

where $\mathbf{X}$ is often called a similarity, and (1) a similarity decomposition.

The maximal set of mutually similar matrices constitute a similarity class of matrices. The algebraic properties which they have in common are called similarity invariants. The principal invariant is the characteristic polynomial $p_{A}(\lambda)=0$, which further implies the invariants

$$
\begin{aligned}
\operatorname{trace}(\mathbf{A}) & =\operatorname{trace}(\mathbf{B}) \\
\operatorname{det}(\mathbf{A}) & =\operatorname{det}(\mathbf{B}) \\
\operatorname{spec}(\mathbf{A}) & =\operatorname{spec}(\mathbf{B})
\end{aligned}
$$

Hence, similarity preserves not only the eigenvalues but it preserves their multiplicities as well.

The Jordan matrix $\mathbf{J}$ to be introduced in the next section is a canonically defined representative of a 
similarity class, which shows all the similarity properties at a glance.

\subsection{Real Jordan decomposition}

From a purely algebraic point of view, the Jordan normal form is a canonical form of similar matrices, i.e., two matrices are similar if they have the same Jordan matrix $\mathbf{J}$ [16]. The Jordan normal form of a matrix is computed by applying a series of similarity transforms $\boldsymbol{\Sigma}_{i}$ to the original matrix $\mathbf{A}$ in order to obtain the Jordan decomposition of the matrix:

$$
\mathbf{A}=\boldsymbol{\Sigma}^{-1} \mathbf{J} \boldsymbol{\Sigma}, \text { where } \boldsymbol{\Sigma}=\boldsymbol{\Sigma}_{1} \boldsymbol{\Sigma}_{2}, \cdots .
$$

The Jordan matrix is a quasi-diagonal matrix, i.e., it is block-diagonal with Jordan blocks $\mathbf{J}_{k}(\lambda)$ that are upper-tridiagonal having the eigenvalues of $\mathbf{A}$ on the diagonal, counting multiplicities, having possibly ones on the super-diagonal, and zeros elsewhere. Hence, a Jordan block has the form:

$$
\mathbf{J}_{k}(\lambda)=\left[\begin{array}{cccc}
\lambda & 1 & 0 & 0 \\
0 & \ddots & \ddots & 0 \\
0 & 0 & \ddots & 1 \\
0 & 0 & 0 & \lambda
\end{array}\right]
$$

In the general case, a complex-conjugate pair of eigenvalues may occur. However, if $\mathbf{A}$ is real, a real Jordan decomposition can be calculated, that replaces the complex conjugate pair by a plane rotation, e.g.,

$$
\left[\begin{array}{cc}
\cos \theta & -\sin \theta \\
\sin \theta & \cos \theta
\end{array}\right]
$$

such that both $\mathbf{J}$ and $\boldsymbol{\Sigma}$ become real matrices.

However the real Jordan decomposition itself is not canonical, i.e., there is a multitude of similarities $\boldsymbol{\Sigma}$ that equally decompose $\mathbf{A}$ into $\mathbf{J}$. The class of matrices $\mathbf{C}$ that commutes with $\mathbf{J}$ completely characterizes this ambiguity. In fact, $\mathbf{C} \boldsymbol{J}=\boldsymbol{J} \mathbf{C}$ gives all possible real Jordan decompositions:

$$
\begin{aligned}
\boldsymbol{\Sigma}^{-1} \mathbf{J} \boldsymbol{\Sigma} & =\boldsymbol{\Sigma}^{-1} \mathbf{J} \mathbf{C}^{-1} \mathbf{C} \boldsymbol{\Sigma} \\
& =\boldsymbol{\Sigma}^{-1} \mathbf{C}^{-1} \mathbf{J C} \boldsymbol{\Sigma} \\
& =(\mathbf{C} \boldsymbol{\Sigma})^{-1} \mathbf{J}(\mathbf{C} \boldsymbol{\Sigma})
\end{aligned}
$$

\subsection{Real Jordan decomposition of displacements}

A rigid transformation is composed of a rotation matrix and a translation vector and it writes as the $4 \times 4$ matrix $\mathbf{D}$. Such a matrix has as eigenvalues $\lambda \in\left\{e^{i \theta}, e^{-i \theta}, 1,1\right\}$ where $\theta$ is the angle of rotation. Therefore there exists a displacement $\boldsymbol{\Sigma}$ such that we obtain a real Jordan decomposition of $\mathbf{D}$ :

$$
\mathbf{D}=\boldsymbol{\Sigma} \mathbf{J} \boldsymbol{\Sigma}^{-1}
$$

with:

$$
\mathbf{J}=\left[\begin{array}{cccc}
\cos \theta & -\sin \theta & 0 & 0 \\
\sin \theta & \cos \theta & 0 & 0 \\
0 & 0 & 1 & \varepsilon \\
0 & 0 & 0 & 1
\end{array}\right]
$$

A displacement $\mathbf{D}$ can be of three different types: a general screw motion, a pure rotation, or a pure translation.

- General motions are a motions that are neither planar motions (or pure rotations) nor pure translations. Their real Jordan factorization is characterized by $\varepsilon=1$. The algebraic multiplicity of $\lambda=1$ is equal to 2 and its geometric multiplicity is equal to 1 [12]. The real Jordan form associated with general motions is:

$$
\mathbf{J}_{R T}=\left[\begin{array}{rrrr}
\cos \theta & -\sin \theta & 0 & 0 \\
\sin \theta & \cos \theta & 0 & 0 \\
0 & 0 & 1 & 1 \\
0 & 0 & 0 & 1
\end{array}\right]
$$

For a general motion, the commuting matrix C defined by eq. (7) has the following form:

$$
\mathbf{C}_{R T}=\left[\begin{array}{rrrr}
a & -b & 0 & 0 \\
b & a & 0 & 0 \\
0 & 0 & c & d \\
0 & 0 & 0 & f
\end{array}\right]
$$

- Pure rotations are characterized by $\varepsilon=0$. The algebraic multiplicity of $\lambda=1$ is equal to 2 and its geometric multiplicity is equal to 2 [1]. It is worthwhile to notice that a planar motion has the same real Jordan factorization as a pure 
rotation:

$$
\mathbf{J}_{R}=\left[\begin{array}{rrrr}
\cos \theta & -\sin \theta & 0 & 0 \\
\sin \theta & \cos \theta & 0 & 0 \\
0 & 0 & 1 & 0 \\
0 & 0 & 0 & 1
\end{array}\right]
$$

In this case the commuting matrix $\mathbf{C}$ has the following form:

$$
\mathbf{C}_{R}=\left[\begin{array}{cccc}
a & -b & 0 & 0 \\
b & a & 0 & 0 \\
0 & 0 & c & d \\
0 & 0 & e & f
\end{array}\right]
$$

- Pure translations are characterized by a null rotation angle, $\theta=0$ and $\varepsilon=1$. For such a motion the algebraic multiplicity of the unique eigenvalue $\lambda=1$ is equal to 4 and its geometric multiplicity is equal to 3 [24]:

$$
\mathbf{J}_{T}=\left[\begin{array}{llll}
1 & 0 & 0 & 0 \\
0 & 1 & 0 & 0 \\
0 & 0 & 1 & 1 \\
0 & 0 & 0 & 1
\end{array}\right] .
$$

Finally, the commuting matrix $\mathbf{C}$ has the following form in this case:

$$
\mathbf{C}_{T}=\left[\begin{array}{cccc}
c_{1} & c_{4} & 0 & c_{7} \\
c_{2} & c_{5} & 0 & c_{8} \\
c_{3} & c_{6} & a & c_{9} \\
0 & 0 & 0 & a
\end{array}\right]
$$

\section{Projective reconstruction with a stereo camera pair}

A pinhole camera projects a point $\boldsymbol{M}$ in 3 -D projective space onto a point $\boldsymbol{m}$ of the 2-D projective plane [6]. This projection can be written as a $3 \times 4$ homogeneous matrix $\mathbf{P}$ of rank equal to 3 :

$$
\mu \boldsymbol{m}=\mathbf{P} \boldsymbol{M}
$$

In this formula $\mu$ is an arbitrary non-null scalar a scale factor. A stereo rig is composed of two cameras fixed together. Let $\mathbf{P}$ and $\mathbf{P}^{\prime}$ be the projection matrices of the left and right cameras.

In this paper we consider uncalibrated cameras, only. It is well known that the epipolar geometry associated with a pair of uncalibrated cameras can be recovered from point-to-point matches between the two images without any knowledge about the 3 -D layout [20], [27]. Moreover, the structure of the projection matrices is, in this case:

$$
\begin{aligned}
\mathbf{P} & =\left[\begin{array}{ll}
\mathbf{I} & \mathbf{0}
\end{array}\right] \\
\mathbf{P}^{\prime} & =\left[\begin{array}{ll}
\overline{\mathbf{P}}^{\prime} & p^{\prime}
\end{array}\right]
\end{aligned}
$$

where $\mathbf{I}$ is the $3 \times 3$ identity matrix, $\overline{\mathbf{P}}^{\prime}$ is a $3 \times 3$ full rank matrix and $\boldsymbol{p}^{\prime}$ is a 3 -vector.

It is possible to compute the $3-\mathrm{D}$ projective coordinates of a point $\boldsymbol{M}$ from the equations $\mu \boldsymbol{m}=$ $\mathbf{P} \boldsymbol{M}$ and $\mu^{\prime} \boldsymbol{m}^{\prime}=\mathbf{P}^{\prime} \boldsymbol{M}$, where $\boldsymbol{m}$ and $\boldsymbol{m}^{\prime}$ are the projections of $\boldsymbol{M}$ onto the left and right images and $\mu$ and $\mu^{\prime}$ are two unknown scale factors:

$$
\left[\begin{array}{ccc}
\mathbf{P} & \boldsymbol{m} & \mathbf{0} \\
\mathbf{P}^{\prime} & \mathbf{0} & \boldsymbol{m}^{\prime}
\end{array}\right]\left[\begin{array}{c}
\mathbf{M} \\
\mu \\
\mu^{\prime}
\end{array}\right]=\mathbf{0}
$$

Equation (18) allows one to compute the projective coordinates $M$ of a $3-\mathrm{D}$ point $M$ in a sensorcentered projective basis. A 3-D projective basis is defined by 5 points in general position. In [13] we show that these points can be chosen such that they are physically attached to the stereo device. Therefore, the projective basis is rigidly moving with the stereo device.

Moreover, since the stereo device is a rigid body, one may attach an Euclidean frame to this body. Let $\boldsymbol{N}$ be the Euclidean homogeneous coordinates of the point $M$. The projective and Euclidean coordinates of the same point $M$ are related by:

$$
\boldsymbol{N} \simeq \mathbf{H}_{P E} \boldsymbol{M}
$$

Matrix $\mathbf{H}_{P E}$ encapsulates both the upgrade of projective space to affine, and the upgrade of affine space to Euclidean space. Namely, eq. (19) takes the projective coordinates of a physical point $M$ and transforms them into $\boldsymbol{N}=\left(\begin{array}{lll}X Y Z T & Y\end{array}\right)^{\top}$. Then the ratios $\left(\frac{X}{T} \frac{Y}{T} \frac{Z}{T}\right)$ are its Cartesian coordinates. The estimation of the entries of $\mathbf{H}_{P E}$ is therefore equivalent to mapping a projective representation into a metric one. Various authors [8], [3], [12] characterized this matrix which can be written as:

$$
\mathbf{H}_{P E}=\left[\begin{array}{rr}
\mathbf{K}^{-1} & \mathbf{0} \\
\boldsymbol{\pi}_{\infty}^{\top} &
\end{array}\right]
$$


In this formula $\mathbf{K}$ is a $3 \times 3$ upper triangular matrix (the internal parameters of the left camera) and $\boldsymbol{\pi}_{\infty}$ is a 4 -vector (the plane of infinity in the projective basis associated with the stereo pair of cameras).

It is however of interest to study projective displacements without explicitin the projective-toEuclidean upgrade $\mathbf{H}_{P E}$, i.e., without computing numerical values for the entries of this matrix.

\section{Projective displacements}

\subsection{Projective reconstruction and rigid motion}

In this section we establish the link between projective reconstruction and rigid motion. First we consider a stereo camera pair (a stereo rig) moving from position 1 to position 2 while it observes a fixed scene. Second we consider a fixed stereo rig observing a rigid object moving from position 1 to position 2. We show that these two situations (moving sensor versus moving object) are similar in a mathematical sense.

Let the stereo rig move from position 1 to position 2. Under the hypotheses that (i) the internal parameters of both cameras are fixed but unknown and that (ii) the spatial relationship between the two cameras is fixed but unknown as well, the stereo rig may be viewed as a moving rigid body. Therefore matrix $\mathbf{H}_{P E}$ of eq. (19) remains the same for any position $i$ and hence the projective displacement $\mathbf{H}_{12}\left(\boldsymbol{M}_{2}=\mathbf{H}_{12} \boldsymbol{M}_{1}\right)$ is related to the rigid displacement $\mathbf{D}_{12}\left(\boldsymbol{N}_{2}=\mathbf{D}_{12} \boldsymbol{N}_{1}\right)$ by the formula $[29,12]$ :

$$
\mathbf{H}_{12}=\alpha \mathbf{H}_{P E}^{-1} \mathbf{D}_{12} \mathbf{H}_{P E}
$$

where $\alpha$ is a non-null scale factor.

So far we considered a moving sensor observing a static scene or a static object. However, in many practical situations, such as the case of a sensor observing a robot manipulator, it is convenient to consider the case of a fixed sensor observing a moving object. Let $\mathbf{D}_{12}^{\circ}$ be the transformation describing

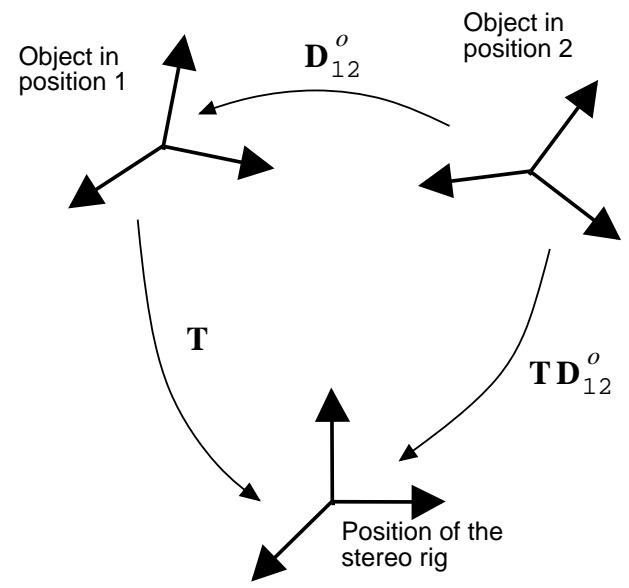

Figure 1: The motion of an object from position 1 to position 2 with respect to a static stereo rig.

the motion of the object, i.e., the object in position 2 with respect to the object in position 1.

Let $\mathbf{T}$ be the rigid transformation describing the position 1 of the object relative to the sensor, Figure 1. If we combine the object's motion with the object's relative position with respect to the sensor we get the relationship between the object in position 2 and the sensor:

$$
\mathbf{T D}_{12}^{\circ}
$$

In order to determine the relationship between the apparent sensor motion and the true object motion, let the object remain fixed and let the sensor move, Figure 2. Let $\mathbf{D}_{12}$ be the transformation describing the apparent sensor motion. The relationship between the object in position 1 and the sensor in its apparent position is:

$$
\mathbf{D}_{12}^{-1} \mathbf{T}
$$

The condition that the apparent sensor motion "absorbs" the real object motion writes:

$$
\mathbf{T D}_{12}^{\circ}=\mathbf{D}_{12}^{-1} \mathbf{T}
$$

We obtain:

$$
\mathbf{D}_{12}=\mathbf{T}^{-1}\left(\mathbf{D}_{12}^{\circ}\right)^{-1} \mathbf{T} .
$$

By combining eqs. (21) and (22) we obtain a similarity relationship between the projective change 


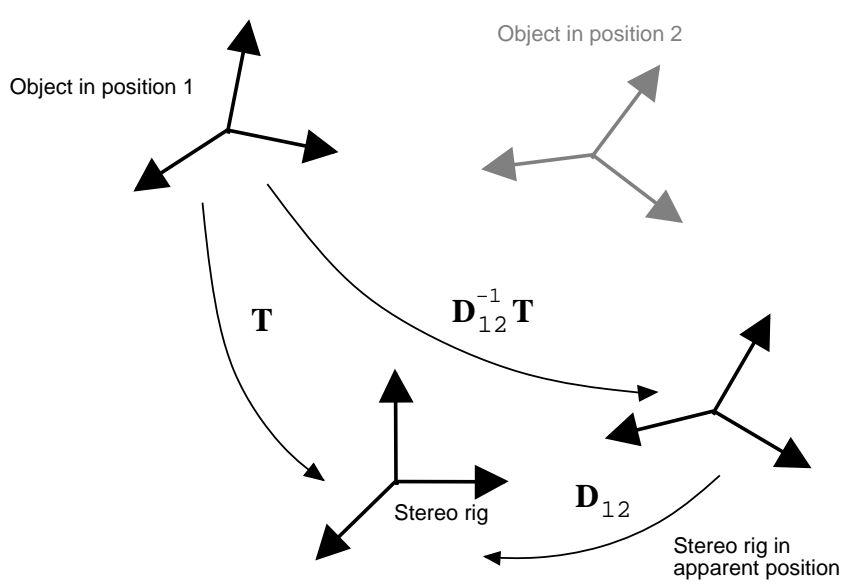

Figure 2: The apparent motion of a stereo rig must be such that the relative position of the object with respect to the sensor remains the same.

of coordinates $\mathbf{H}_{12}$ and the motion of an object observed with a static stereo rig:

$$
\mathbf{H}_{12}=\alpha\left(\mathbf{T H}_{P E}\right)^{-1}\left(\mathbf{D}_{12}^{\circ}\right)^{-1} \mathbf{T} \mathbf{H}_{P E} .
$$

\subsection{The Lie group of projective dis- placements}

The analysis performed above allows us, regardless of whether the stereo rig or the observed object is moving, to relate a projective transformation to a rigid transformation:

$$
\mathbf{H}=\alpha \mathbf{H}_{P E}^{-1} \mathbf{D} \mathbf{H}_{P E}
$$

The fact that traces and determinants are invariant under similarity transformations allows us to compute the scale factor $\alpha$ and define a normalized projective displacement. Indeed from equations (2) and (3) we have:

$$
\operatorname{trace}(\mathbf{H})=\operatorname{trace}(\alpha \mathbf{D})=2 \alpha(1+\cos \theta)
$$

and

$$
\operatorname{det}(\mathbf{H})=\operatorname{det}(\alpha \mathbf{D})=\alpha^{4}
$$

where $\theta$ is the angle of rotation associated with the displacement D. Since we have $1+\cos \theta \geq 0$ we obtain:

$$
\operatorname{sign}(\alpha)=\operatorname{sign}(\operatorname{trace}(\mathbf{H}))
$$

Therefore, the scale factor $\alpha$ can be uniquely determined for every homography $\mathbf{H}$ that is conjugated to a displacement:

$$
\alpha=\operatorname{sign}(\operatorname{trace}(\mathbf{H})) \sqrt[4]{\operatorname{det}(\mathbf{H})}
$$

From now on, we replace the homogeneous homography by a normalized homography:

$$
\mathbf{H}_{R T}=\frac{1}{\alpha} \mathbf{H}=\mathbf{H}_{P E}^{-1} \mathbf{D} \mathbf{H}_{P E}
$$

$\mathbf{H}_{R T}$ will be referred to as a projective displacement or projective motion because it describes the motion of a rigid body observed with an uncalibrated stereo rig. It is well known that the $4 \times 4$ homographies form the projective group PGL(3). It will be shown that, under certain restrictions, the projective displacements - the matrices of the form of $\mathbf{H}_{R T}$ - form a sub-group of the projective group. The algebraic and differential properties of this group will allow us to characterize rigid body motion in a non-metric space.

Under the assumption that $\mathbf{H}_{P E}$ remains constant over time we immediately obtain the following properties:

1. The product of two projective displacements $\mathbf{H}_{R T 1}$ and $\mathbf{H}_{R T 2}$ is a projective displacement as well:

$$
\mathbf{H}_{R T 1} \mathbf{H}_{R T 2}=\mathbf{H}_{P E}^{-1} \mathbf{D}_{1} \mathbf{H}_{P E} \mathbf{H}_{P E}^{-1} \mathbf{D}_{2} \mathbf{H}_{P E}=\mathbf{H}_{P E}^{-1} \mathbf{D}_{1} \mathbf{D}_{2} \mathbf{H}_{P E}
$$

2. The identity matrix is a projective displacement, and

3. The inverse of a projective displacement is a projective displacement:

$$
\left(\mathbf{H}_{R T}\right)^{-1}=\mathbf{H}_{P E}^{-1} \mathbf{D}^{-1} \mathbf{H}_{P E}
$$

The group of projective displacements is isomorphic to the group of rigid displacements. Since the displacement group is a Lie group, we conclude that the group of projective displacements is a Lie group as well.

Finally, it is possible to compute the tangent operator on the group of projective displacements:

$$
\frac{d \mathbf{H}_{R T}(t)}{d t}\left(\mathbf{H}_{R T}\right)^{-1}=\mathbf{H}_{P E}^{-1} \frac{d \mathbf{D}^{-1}(t)}{d t} \mathbf{D}^{-1} \mathbf{H}_{P E}
$$




\subsection{Orbit of rigidly moving points and their height}

In this section, we characterize the action of projective displacements onto the projective coordinates of a point. First, we characterize the scale or orbital height of projectively reconstructed points. Second, we show that this height remains invariant under projective displacements. Finally, we show that the orbit of the projective coordinates of a point under action of projective displacements lies within a hy-

Equation (19) relates the projective coordinates $\boldsymbol{M}$ of a point to its homogeneous Euclidean coordinates $\boldsymbol{N}$. The actual coordinate vector of the plane at infinity $\boldsymbol{\pi}_{\infty}$ in $\mathbf{H}_{P E}$ (20) defines the height $\rho$ of $\boldsymbol{N}$ that is used to calculate the normalized coordinates $\boldsymbol{X}=(X, Y, Z, 1)^{T}$.

$$
\rho=\pi_{\infty}^{\top} \boldsymbol{M}, \quad \boldsymbol{X}=1 / \rho \boldsymbol{N}=1 / \rho \mathbf{H}_{P E} \boldsymbol{M} .
$$

Since in the uncalibrated case the upgrade is supposed not to be known, so is the height of a point. However, $\rho$ is implicitly associated with the $\mathbb{R}^{4}$ vector $\boldsymbol{M}_{\rho}$ calculated during the reconstruction. We distinguish this fix $\mathbb{R}^{4}$-vector from the general homogeneous coordinate vector $\boldsymbol{M}$ by indexing it with $\rho$, which is an individual property of each point-vector.

$$
\boldsymbol{M} \simeq \boldsymbol{M}_{\rho}=\rho \mathbf{H}_{P E}^{-1} \boldsymbol{X}
$$

Equation (27) shows that $\boldsymbol{M}_{\rho}$ is linear in the three parameters, $X, Y, Z$, thus point-vectors with the same height define a hyperplane in $\mathbb{R}^{4}$ with equation $\pi_{\infty}^{\top} \boldsymbol{M}_{\rho}=\rho$.

Given now a point-vector $\boldsymbol{M}_{\rho}$, its orbit under action of the group of projective displacements $\mathbf{H}_{R T}$ is defined as:

$$
\mathcal{M}_{\rho}=\left\{\mathbf{H}_{R T} \boldsymbol{M}_{\rho} \mid \text { for all } \mathbf{H}_{R T}\right\},
$$

Such an orbit lies entirely within in a hyperplane of $\mathbb{R}^{4}$. Let $\boldsymbol{M}_{\rho}^{\prime} \in \mathcal{M}_{\rho}$. Hence $\boldsymbol{M}_{\rho}^{\prime}=\mathbf{H}_{R T} \boldsymbol{M}_{\rho}$ and (with $\boldsymbol{X}^{\prime}=\mathbf{D} \boldsymbol{X}$ ) we obtain: perplane of $\mathbb{R}^{4}$.

called the orbital height of an entire hyperplane orbit.

Figure 3 illustrates the trajectory of a pointvector in $\mathcal{M}_{\rho}$. This trajectory (or orbit) lies in a 3-D subspace illustrated by a box, which is nothing else than the above hyperplane with height $x_{4}=\rho$.

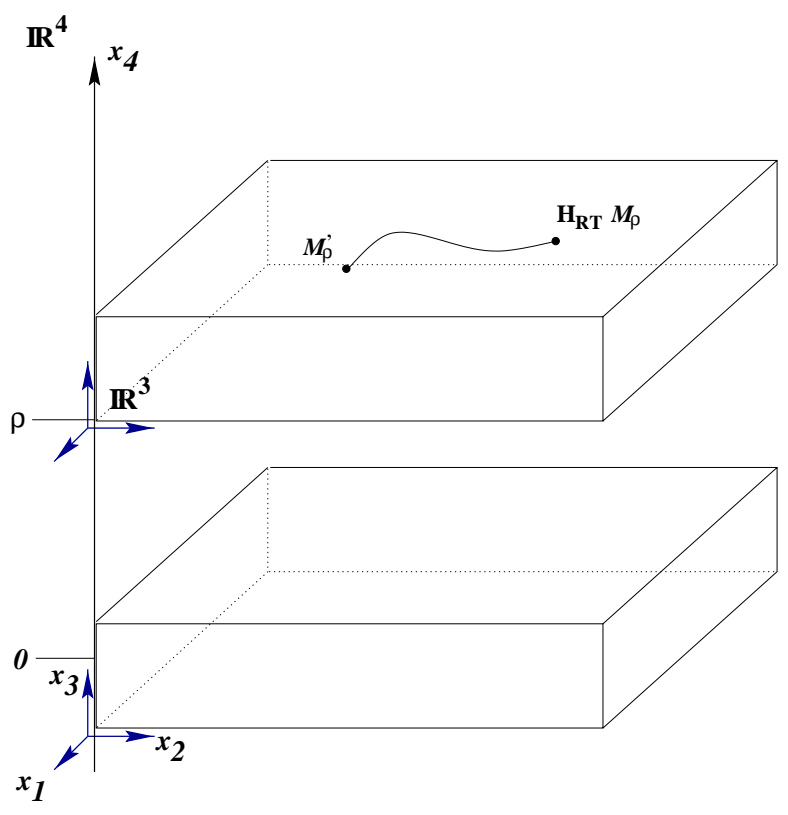

Figure 3: The trajectory of $\boldsymbol{M}_{\rho}$ in a $\mathbb{R}^{4}$-hyperplane. The box illustrates such a hyperplane as 3D space with $x_{1}, x_{2}, x_{3}$. The height of this hyperplane above the origin is $x_{4}=\rho$. It is also the orbital height of $\boldsymbol{M}_{\rho}$ under the action of projective displacements.

\subsection{Non-metric linear spatial veloc- ity}

In this section, we show $\frac{d}{d t} \boldsymbol{M}_{\rho}(t)=\dot{\boldsymbol{M}}_{\rho}(t)$ to be the linear velocity of a rigidly moving point $M$ in projective space restricted to an orbit $\rho$. It corresponds to the metric linear velocity of $M$ is defined as the temporal derivative of its coordinate vector $\dot{X}$.

Consider the position of a moving point $M$ at $\boldsymbol{M}_{\rho}^{\prime}=\mathbf{H}_{R T} \boldsymbol{M}_{\rho}=\mathbf{H}_{P E}^{-1} \mathbf{D} \mathbf{H}_{P E} \boldsymbol{M}_{\rho}=\rho \mathbf{H}_{P E}^{-1} \mathbf{D} \boldsymbol{X}=d \dot{\mathbf{H}_{P} e_{E}^{1}}$ OXdnd at time $t$. We have the following relaTherefore the group of projective displacements leaves the height of a point invariant, and $\rho$ is also tionship:

$$
\boldsymbol{M}_{\rho}(t)=\mathbf{H}_{R T}(t) \boldsymbol{M}_{\rho}(0)
$$


By combining this formula with equations (25) and (28) we obtain:

$$
\begin{aligned}
\boldsymbol{M}_{\rho}(t) & =\mathbf{H}_{R T}(t) \boldsymbol{M}_{\rho}(0) \\
& =\mathbf{H}_{P E}^{-1} \mathbf{D}(t) \mathbf{H}_{P E} \rho \mathbf{H}_{P E}^{-1} \boldsymbol{X}(0) \\
& =\rho \mathbf{H}_{P E}^{-1} \mathbf{D}(t) \boldsymbol{X}(0) \\
& =\rho \mathbf{H}_{P E}^{-1} \boldsymbol{X}(t)
\end{aligned}
$$

Hence, we obtain:

$$
\dot{\boldsymbol{M}}_{\rho}(t)=\rho \mathbf{H}_{P E}^{-1} \dot{\boldsymbol{X}}
$$

In summary, $\dot{\boldsymbol{M}}_{\rho}$ is a non-metric point velocity in projective space. It is related to the corresponding metric velocity $\dot{\boldsymbol{X}}$ by equation (30). The nonmetric velocity is defined only up to an unknown scale factor $\rho$, different for each individual point.

\section{Projective translations}

In this section we are going to study the projective representation of pure translations. By combining eq. (5) with eq. (25) and by considering the real Jordan form of a pure translation given by eq. (13) we obtain:

$$
\mathbf{H}_{T}=\mathbf{H}_{P E}^{-1} \boldsymbol{\Sigma}^{-1} \mathbf{J}_{T} \boldsymbol{\Sigma} \mathbf{H}_{P E}=\mathbf{H}_{J}^{-1} \mathbf{J}_{T} \mathbf{H}_{J}
$$

where $\mathbf{H}_{J}=\boldsymbol{\Sigma} \mathbf{H}_{P E}$ is a $4 \times 4$ homography. Since the real Jordan decomposition is not unique, the above decomposition is not unique and hence there exists a full rank matrix $\mathbf{C}_{T}$ which verifies $\mathbf{H}_{J} \mathbf{C}_{T}=$ $\mathbf{C}_{T} \mathbf{H}_{J}$ where $\mathbf{C}_{T}$ is given by eq. (14).

Below we derive a unique parameterization of projective translations and we show that, under certain constraints, projective translations build a Lie group.

\subsection{Parameterization of projective translations}

In this section we devise a parameterization of projective translations in terms of a magnitude $s$ and a $4 \times 4$ matrix of rank equal to 1 . We show that, unlike the real Jordan decomposition outlined above, this parameterization is unique.
Proposition 1 A projective translation is a projective transformation that is conjugated to a pure translation. The $4 \times 4$ homography describing this transformation can be parameterized as:

$$
\mathbf{H}_{T}(s)=\mathbf{I}+s \hat{\mathbf{H}}_{t}
$$

This parameterization is unique.

Proof: In order to prove this proposition let us consider matrix $\mathbf{H}_{J}$ defined above and its inverse. The row vectors of $\mathbf{H}_{J}$ are denoted by $\boldsymbol{h}_{i}^{\top}$ and the columns of $\mathbf{H}_{J}^{-1}$ are denoted by $\boldsymbol{k}_{i}$. Therefore we have:

$$
\boldsymbol{h}_{i}^{\top} \boldsymbol{k}_{j}=\boldsymbol{k}_{j}^{\top} \boldsymbol{h}_{i}=\delta_{i j}
$$

where $\delta_{i j}$ is equal to 1 if $i=j$ and is equal to 0 if $i \neq j$. By subtracting the identity matrix from $\mathbf{J}_{T}$ and pulling it out from the similarity relation in eq. (31) we have:

$$
\begin{aligned}
& \mathbf{H}_{T}=\underbrace{\left[\begin{array}{llll}
\boldsymbol{k}_{1} & \boldsymbol{k}_{2} & \boldsymbol{k}_{3} & \boldsymbol{k}_{4}
\end{array}\right]}_{\mathbf{H}_{\boldsymbol{J}}^{-1}} \underbrace{\left[\begin{array}{llll}
0 & 0 & 0 & 0 \\
0 & 0 & 0 & 0 \\
0 & 0 & 0 & 1 \\
0 & 0 & 0 & 0
\end{array}\right]}_{\mathbf{J}_{T}-\mathbf{I}} \underbrace{\left[\begin{array}{l}
\boldsymbol{h}_{1}^{\top} \\
\boldsymbol{h}_{2}^{\top} \\
\boldsymbol{h}_{3}^{\top} \\
\boldsymbol{h}_{4}^{\top}
\end{array}\right]}_{\mathbf{H}_{\boldsymbol{J}}}+\underbrace{\left[\begin{array}{llll}
1 & 0 & 0 & 0 \\
0 & 1 & 0 & 0 \\
0 & 0 & 1 & 0 \\
0 & 0 & 0 & 1
\end{array}\right]}_{\mathbf{I}} \\
& =\boldsymbol{k}_{3} \boldsymbol{h}_{4}^{\top}+\mathbf{I}=\mathbf{I}+\hat{\mathbf{H}}_{t}
\end{aligned}
$$

Matrix $\hat{\mathbf{H}}_{t}=\boldsymbol{k}_{3} \boldsymbol{h}_{4}^{\top}$ has rank equal to 1 , trace equal to 0 , and is nilpotent with order $1: \hat{\mathbf{H}}_{t}^{2}=$ $\boldsymbol{k}_{3} \boldsymbol{h}_{4}^{\top} \boldsymbol{k}_{3} \boldsymbol{h}_{4}^{\top}=\boldsymbol{k}_{3} \delta_{34} \boldsymbol{h}_{4}^{\top}=0$. - q.e.d.

$\hat{\mathbf{H}}_{t}$ is called the generator of the projective translation. A normalized generator can be defined such that $\hat{\mathbf{H}}_{t}=s \hat{\mathbf{H}}_{t}^{\prime}$ with $s=\left\|\boldsymbol{h}_{4}\right\|\left\|\boldsymbol{k}_{3}\right\|$. Therefore we obtain the following parameterization for the projective translation:

$$
\mathbf{H}_{T}(s)=\mathbf{I}+s \hat{\mathbf{H}}_{t} .
$$

Finally we show that this parameterization is unique. Consider two real Jordan decomposition of a projective translation, $\mathbf{H}_{J}^{-1} \mathbf{J}_{T} \mathbf{H}_{J}=\mathbf{H}_{J}^{\prime-1} \mathbf{J}_{T} \mathbf{H}^{\prime}{ }_{J}$ with $\mathbf{H}_{J}^{\prime}=\mathbf{H}_{J} \mathbf{C}_{T}$. We must have:

$$
\mathbf{H}_{T}=\mathbf{I}+\boldsymbol{k}_{3} \boldsymbol{h}_{4}^{\top}=\mathbf{I}+\boldsymbol{k}_{3}^{\prime} \boldsymbol{h}_{4}^{\prime \top}
$$

from which we get that $\boldsymbol{k}_{3}^{\prime}=1 / a \boldsymbol{k}_{3}$ and $\boldsymbol{h}_{4}^{\prime}=a \boldsymbol{h}_{4}$. Therefore the matrix generator $\hat{\mathbf{H}}_{t}$ is the same for both decompositions. 


\subsection{The Lie group of projective 6 Projective rotations translations}

Proposition 2 The projective translations $\mathbf{H}_{T}(s)$

In this section we are going to study the projecwith a fixed normalized generator $\hat{\mathbf{H}}_{t}$ build a Lie group.

tive representation of pure rotations. By combining eq. (5) with eq. (25) and by considering the real Jordan form of a pure rotation given by eq. (11) we obtain:

Proof: First, it is straightforward to show that the projective translations with a fixed normalized generator form a group:

$$
\mathbf{H}_{R}=\mathbf{H}_{P E}^{-1} \boldsymbol{\Sigma}^{-1} \mathbf{J}_{R} \boldsymbol{\Sigma} \mathbf{H}_{P E}=\mathbf{H}_{J}^{-1} \mathbf{J}_{R} \mathbf{H}_{J}
$$

where $\mathbf{H}_{J}$ is a $4 \times 4$ homography. Since the real $\begin{array}{llll} & \mathbf{H}_{T}(0) & =\mathbf{I} & \text { Jordan decomposition is not unique, the above de- } \\ \text { identity: } & \mathbf{H}_{T}\left(s_{1}\right) & \mathbf{H}_{T}\left(s_{2}\right) & =\mathbf{I}+s_{1} \hat{\mathbf{H}}_{t}+s_{2} \mathbf{H}_{t} \mathbf{H}_{s_{1}} s_{2} \mathbf{H}_{t}^{2 i} \text { not unique and hence there exists a } \\ \text { closure: } & & \end{array}$ closure: $\quad \mathbf{H}_{T}\left(s_{1}\right) \quad \mathbf{H}_{T}\left(s_{2}\right)=\mathbf{I}+s_{1} \hat{\mathbf{H}}_{t}+s_{2} \mathbf{H}_{t}{ }_{s} s_{2} \mathbf{H}_{t}^{2} \mathbf{H}_{R}$ which verifies $\mathbf{H}_{J} \mathbf{C}_{R}=\mathbf{C}_{R} \mathbf{H}_{J}$ commutative: $\begin{array}{lll}\mathbf{H}_{T}\left(s_{2}\right) \quad \mathbf{H}_{T}\left(s_{1}\right)=\mathbf{I}+\left(s_{1}+s_{2}\right) \mathbf{H}_{t} & =\mathbf{I}\end{array}$ inverse: $\quad \mathbf{H}_{T}(s) \quad \mathbf{H}_{T}(-s)=\mathbf{I}+(s-s) \hat{\mathbf{H}}_{t}=\mathbf{A}$ s with projective translations, we derive a paIt is a continuous, one-dimensional group in the variable $s$. There exists a corresponding manifold $\Phi$ which is linear, hence continuous, differentiable, hence smooth:

$$
\Phi: \mathbb{R} \rightarrow \mathbb{R}^{4 \times 4}: \mathbf{H}_{T}(s)=\mathbf{I}+s \hat{\mathbf{H}}_{t},
$$

It is a group strictly isomorphic to the additive group $\mathbb{R}^{1}$. The limit in vicinity of the identity yields the tangent to this group

$$
\lim _{s \rightarrow 0} \frac{\mathbf{H}_{T}(s)-\mathbf{I}}{s}=\hat{\mathbf{H}}_{t}
$$

which is a matrix representation of the corresponding Lie algebra. The corresponding Lie brackets are trivially $\left[a \hat{\mathbf{H}}_{t}, b \hat{\mathbf{H}}_{t}\right]=0$. - q.e.d.

It can be verified using the nil-potency of $\hat{\mathbf{H}}_{t}$ that the matrix exponential is a bijection from the Lie algebra onto the Lie group:

$\exp \left(s \hat{\mathbf{H}}_{t}\right)=\sum_{k=0}^{\infty} \frac{\left(s \hat{\mathbf{H}}_{t}\right)^{k}}{k !}=\mathbf{I}+s \hat{\mathbf{H}}_{t}+0+0 \cdots=\mathbf{H}_{T}(s)$

Its inverse, i.e., a matrix logarithm from the Lie group to the Lie algebra is calculated by:

$$
s \hat{\mathbf{H}}_{t}=\mathbf{H}_{T}-\mathbf{I}
$$

or by:

$$
s \hat{\mathbf{H}}_{t}=\frac{1}{2}\left(\mathbf{H}_{T}(s)-\mathbf{H}_{T}^{-1}(s)\right)
$$

Proof: Eq. (39) writes:

. Interestingly enough, this parameterization is a generalization of the well known Rodriguez' formula for parameterizing $3 \times 3$ matrices associated with rotations in Euclidean space.

\subsection{Rodriguez formula for projective rotations}

Proposition 3 A projective rotation is a projective transformation that is conjugated to a pure rotation. The $4 \times 4$ homography describing this transformation can be parameterized as:

$$
\mathbf{H}_{R}(\theta)=\mathbf{I}+\sin \theta \hat{\mathbf{H}}_{r}+(1-\cos \theta) \hat{\mathbf{H}}_{r}^{2}
$$

This parameterization is unique up to the sign of the angle of rotation $\theta$ and it will be referred to as the "Rodriguez formula for projective rotations".

Using the same notations as in the case of translations eq. (32), we obtain:

$$
\begin{aligned}
\mathbf{H}_{R} & =\underbrace{\left[\begin{array}{llll}
\boldsymbol{k}_{1} & \boldsymbol{k}_{2} & \boldsymbol{k}_{3} & \boldsymbol{k}_{4}
\end{array}\right]}_{\mathbf{H}_{J}^{-1}} \underbrace{\left[\begin{array}{cccc}
\cos \theta-1 & -\sin \theta & 0 & 0 \\
\sin \theta & \cos \theta-1 & 0 & 0 \\
0 & 0 & 0 & 0 \\
0 & 0 & 0 & 0
\end{array}\right]}_{\mathbf{J}_{R}-\mathbf{I}} \underbrace{\left[\begin{array}{l}
\boldsymbol{h}_{1}^{\top} \\
\boldsymbol{h}_{2}^{\top} \\
\boldsymbol{h}_{3}^{\top} \\
\boldsymbol{h}_{4}^{\top}
\end{array}\right]}_{\mathbf{H}_{J}} \\
& =(\cos \theta-1)\left(\boldsymbol{k}_{1} \boldsymbol{h}_{1}^{\top}+\boldsymbol{k}_{2} \boldsymbol{h}_{2}^{\top}\right)+\sin \theta\left(\boldsymbol{k}_{2} \boldsymbol{h}_{1}^{\top}-\boldsymbol{k}_{1} \boldsymbol{h}_{2}^{\top}\right)+\mathbf{I}
\end{aligned}
$$


We introduce the following notations:

$$
\hat{\mathbf{H}}_{s}=\boldsymbol{k}_{1} \boldsymbol{h}_{1}^{\top}+\boldsymbol{k}_{2} \boldsymbol{h}_{2}^{\top}
$$

and

$$
\hat{\mathbf{H}}_{r}=\boldsymbol{k}_{2} \boldsymbol{h}_{1}^{\top}-\boldsymbol{k}_{1} \boldsymbol{h}_{2}^{\top}
$$

Remember that the $\boldsymbol{h}_{i}^{\top}$ are the row vectors of $\mathbf{H}_{J}$ and the $\boldsymbol{k}_{i}$ are the column vectors of $\mathbf{H}_{J}^{-1}$. Therefore we have $\boldsymbol{k}_{i}^{\top} \boldsymbol{h}_{j}=\boldsymbol{h}_{j}^{\top} \boldsymbol{k}_{i}=\delta_{i j}$ and we immediately obtain:

$$
\hat{\mathbf{H}}_{s}=-\hat{\mathbf{H}}_{r}^{2} .
$$

Notice that we also have:

$$
\begin{aligned}
\hat{\mathbf{H}}_{s}^{2} & =\hat{\mathbf{H}}_{s}, \\
\hat{\mathbf{H}}_{r} & =\hat{\mathbf{H}}_{s} \hat{\mathbf{H}}_{r} .
\end{aligned}
$$

By induction we obtain:

$$
\begin{aligned}
\hat{\mathbf{H}}_{r}^{2 n+2} & =(-1)^{n} \hat{\mathbf{H}}_{r}^{2}, \\
\hat{\mathbf{H}}_{r}^{2 n+1} & =(-1)^{n} \hat{\mathbf{H}}_{r} .
\end{aligned}
$$

By substituting eqs. (43), (42), and (41) into eq. (40) we obtain the Rodriguez formula for projective rotations:

$$
\mathbf{H}_{R}(\theta)=\mathbf{I}+\sin \theta \hat{\mathbf{H}}_{r}+(1-\cos \theta) \hat{\mathbf{H}}_{r}^{2} .
$$

In order to prove that this parameterization is unique, consider the expression of $\hat{\mathbf{H}}_{r}$ and replace $\mathbf{H}_{J}$ with $\mathbf{C}_{R} \mathbf{H}_{J}$ : (note that $\mathbf{C}_{R}$ commutates)

$$
\begin{aligned}
\hat{\mathbf{H}}_{r} & =\boldsymbol{k}_{2} \boldsymbol{h}_{1}^{\top}-\boldsymbol{k}_{1} \boldsymbol{h}_{2}^{\top} \\
& =\mathbf{H}_{J}^{-1}\left[\begin{array}{cccc}
0 & -1 & 0 & 0 \\
1 & 0 & 0 & 0 \\
0 & 0 & 0 & 0 \\
0 & 0 & 0 & 0
\end{array}\right] \mathbf{H}_{J} \\
& =\mathbf{H}_{J}^{-1} \mathbf{C}_{R}^{-1}\left[\begin{array}{cccc}
0 & -1 & 0 & 0 \\
1 & 0 & 0 & 0 \\
0 & 0 & 0 & 0 \\
0 & 0 & 0 & 0
\end{array}\right] \mathbf{C}_{R} \mathbf{H}_{J} \\
& =\mathbf{H}_{J}^{-1} \mathbf{C}_{R}^{-1} \mathbf{C}_{R}\left[\begin{array}{cccc}
0 & -1 & 0 & 0 \\
1 & 0 & 0 & 0 \\
0 & 0 & 0 & 0 \\
0 & 0 & 0 & 0
\end{array}\right] \mathbf{H}_{J}
\end{aligned}
$$

Hence, matrix $\hat{\mathbf{H}}_{r}$ is the same for all real Jordan decompositions of a projective rotation.

\subsection{The Lie group of projective ro- tations}

Proposition 4 The projective rotations $\mathbf{H}_{R}(\theta)$ with a fixed matrix $\hat{\mathbf{H}}_{r}$ build a Lie group.
Proof: The group axioms are formally verified by using the Rodriguez formula for projective rotations and by applying eqs. (46) and (47):

$$
\begin{array}{lll}
\text { identity: } & & \mathbf{H}_{R}(0)=\mathbf{I} \\
\text { closure: } & \mathbf{H}_{R}\left(\theta_{1}\right) & \mathbf{H}_{R}\left(\theta_{2}\right)=\mathbf{I}+\sin \left(\theta_{1}+\theta_{2}\right) \hat{\mathbf{H}}_{r}+\left(1-\cos \left(\theta_{1}\right.\right. \\
\text { commutative: } & \mathbf{H}_{R}\left(\theta_{2}\right) & \mathbf{H}_{R}\left(\theta_{1}\right)=\mathbf{H}_{R}\left(\theta_{2}+\theta_{1}\right)=\mathbf{H}_{R}\left(\theta_{1}+\theta_{2}\right) \\
\text { inverse: } & \mathbf{H}_{R}(\theta) & \mathbf{H}_{R}(-\theta)=\mathbf{I}
\end{array}
$$

It is a continuous, one-dimensional group in the variable $\theta$. There exists a corresponding smooth manifold $\Phi$ :

$\Phi: \mathbb{R} \rightarrow \mathbb{R}^{4 \times 4}: \mathbf{H}_{R}(\theta)=\mathbf{I}+\sin \theta \hat{\mathbf{H}}_{r}+(1-\cos \theta) \hat{\mathbf{H}}_{r}^{2}$

The tangent in $\mathbb{R}^{4 \times 4}$ to this pure rotation $\mathbf{H}_{R}(\theta)$ in $\theta$ follows from simple differentiation

$$
\frac{d}{d \theta} \mathbf{H}_{R}(\theta)=\cos \theta \hat{\mathbf{H}}_{r}+\sin \theta \hat{\mathbf{H}}_{r}^{2}=\hat{\mathbf{H}}_{r} \mathbf{H}_{R}(\theta) .
$$

The matrix $\hat{\mathbf{H}}_{r}$ is indeed the tangent operator of this group, i.e. the matrix operator that calculates the derivation or motion tangent by simple leftmultiplication with the projective rotation.

Note that eq. (51) is a differential equation whose solution (with $\mathbf{H}_{R}(0)=\mathbf{I}$ ) is the matrix exponential:

$$
\exp \left(\theta \hat{\mathbf{H}}_{r}\right)=\sum_{n=0}^{\infty} \frac{\theta^{n} \hat{\mathbf{H}}_{r}^{n}}{n !}
$$

By using the odd and even powers of matrix $\hat{\mathbf{H}}_{r}$ given by eqs. (46), (47), and the power series of the trigonometric functions we obtain the above Rodriguez formula:

$$
\exp \left(\theta \hat{\mathbf{H}}_{r}\right)=\mathbf{I}+\sin \theta \hat{\mathbf{H}}_{r}+(1-\cos \theta) \hat{\mathbf{H}}_{r}^{2}=\mathbf{H}_{R}(\theta)
$$

Finally, let us show how to derive $\hat{\mathbf{H}}_{r}$ from the homography $\mathbf{H}_{R}$. Remember that the trace of a projective displacement is equal to the trace of a displacement, trace $\left(\mathbf{H}_{R}\right)=2(1+\cos \theta)$. From the Rodriguez formula we obtain:

$$
\mathbf{H}_{R}(\theta)-\mathbf{H}_{R}(-\theta)=2 \sin \theta \hat{\mathbf{H}}_{r}
$$

Hence:

$$
\hat{\mathbf{H}}_{r}=\frac{1}{2 \sin \theta}\left(\mathbf{H}_{R}-\mathbf{H}_{R}^{-1}\right) \text {.- q.e.d. }
$$


Notice that there is a sign ambiguity due to the computation of $\sin \theta$ from the trace of the projective rotation. This ambiguity reflects two possible orientations of the axis of rotation, one orientation associated with $\theta$ and the other orientation associated with $-\theta$.

\section{Projective geometry of a stereo-robot configuration}

So far we studied projective displacements, rigid motion observed by a camera pair in proje tive space. In this section we are interested in mod eling the kinematics of an articulated mechanism such as a robot manipulator in terms of projectiv displacements, namely projective translations and projective rotations. Without loss of generality we consider a robot with revolute joints. As in the previous sections we consider a stereo rig mounted onto an independent fixture. The cameras associated with this stereo rig observe the robot's motions: the robot's end-effector is supposed to lie in the field of view of both cameras. At each position of the end-effector the stereo rig is able to provide projective reconstruction of the end-effector by means of a set of points physically and rigidly attached to the end-effector. Moreover, if the end-effector moves from one position to another position, it is possible to compute the corresponding projective transformation. Since the stereo rig remains fixed and the end-effector moves in front of it, the relationship between the measured projective transformation and the true motion of the end-effector is described by equation (23).

In order to introduce the projective model associated with such a stereo-robot setup and to represent the robot's kinematic map in terms of projective displacements we start by considering the standard Euclidean model. There are three Euclidean frames associated with the setup of Figure 4:

1. a stereo rig frame $\mathcal{E}$,

2. a hand frame $\mathcal{H}$ associated with the endeffector, and

3. a robot base frame $\mathcal{B}$.

Figure 4: The stereo rig observes the robot in various configurations: a zero-configuration and a general configuration characterized by the vector $\boldsymbol{q}$ of joint variables. The Cartesian frame illustrated on this figure ARE NOT used. Instead, the projective approach uses points physically attached to the end-effector and observed by both cameras. 
A robot posture is defined by the transformation $\mathbf{D}_{H B}$ which describes the position of the hand frame $\mathcal{H}$ with respect to the base frame $\mathcal{B}$. Moreover the forward kinematic map of a robot allows one to express this transformation as a function of the robot's link-geometry and its joint-angles:

$$
\mathbf{D}_{H B}(\boldsymbol{q})=\mathbf{D}_{H 6} \mathbf{Q}_{6}\left(\theta_{6}\right) \mathbf{Q}_{5}\left(\theta_{5}\right) \ldots \mathbf{Q}_{1}\left(\theta_{1}\right) \mathbf{D}_{1 B},
$$

Let us make explicit the notations used in this equation. The configuration of a robot with six revolute joints ( 6 degrees of freedom) can be written as a vector of joint variables $\boldsymbol{q}=\left(\theta_{1}, \ldots, \theta_{6}\right)^{T}$ which makes up the robot's joint space. Matrices $\mathbf{Q}_{i}\left(\theta_{i}\right)$ describe the joint action between connected links using the classical Denavit-Hartenberg formulation:

$$
\mathbf{Q}_{i}\left(\theta_{i}\right)=\left[\begin{array}{cccc}
1 & 0 & 0 & r_{i} \\
0 & \cos \alpha_{i} & -\sin \alpha_{i} & 0 \\
0 & \sin \alpha_{i} & \cos \alpha_{i} & l_{i} \\
0 & 0 & 0 & 1
\end{array}\right]\left[\begin{array}{cccc}
\cos \theta_{i} & -\sin \theta_{i} & 0 & 0 \\
\sin \theta_{i} & \cos \theta_{i} & 0 & 0 \\
0 & 0 & 1 & 0 \\
0 & 0 & 0 & 1
\end{array}\right]
$$

where $\alpha_{i}, r_{i}$, and $l_{i}$ are the Denavit-Hartenberg parameters associated with the $i^{\text {th }}$ link. $\mathbf{D}_{H 6}$ and $\mathbf{D}_{B 1}$ are rigid links that allow to deliberately choose the hand- and base frame.

Without loss of generality, the offsets can be chosen for an arbitrary configuration to become the zero-configuration $\boldsymbol{q}=\mathbf{0}$, and $\mathbf{D}_{H B}(\mathbf{0})$ is the corresponding zero-posture of the robot. The displacement of the hand away from the zero configuration defines a relative forward kinematic map as shown on Figure 4:

$$
\mathbf{D}_{H}(\boldsymbol{q})=\mathbf{D}_{H B}(\mathbf{0}) \mathbf{D}_{H B}^{-1}(\boldsymbol{q})
$$

Let us operate each joint $i$ separately and introduce the following notations which simplify the formulae:

$$
\mathbf{R}_{i}\left(\theta_{i}\right)=\mathbf{D}_{H}\left(0, \ldots, \theta_{i}, \ldots, 0\right)
$$

By combining eq. (55) with eq. (57) we obtain for $\mathbf{R}_{i}$ :

$$
\mathbf{R}_{i}\left(\theta_{i}\right)=\mathbf{T}_{i}^{-1} \mathbf{Q}_{i}(0) \mathbf{Q}_{i}^{-1}\left(\theta_{i}\right) \mathbf{T}_{i},
$$

where $\mathbf{T}_{i}=\mathbf{Q}_{i+1}^{-1}(0) \ldots \mathbf{Q}_{6}^{-1}(0) \mathbf{D}_{H 6}^{-1}$. Using eq. (56) it is straightforward to verify that matrix
$\mathbf{Q}_{i}(0) \mathbf{Q}_{i}^{-1}\left(\theta_{i}\right)$ is a pure rotation of angle $\theta_{i}$. Therefore, by the similarity transformation (59), matrix $\mathbf{R}_{i}\left(\theta_{i}\right)$ represents a rotation as well. Finally, by directly multiplying $\mathbf{R}_{1}\left(\theta_{1}\right)$ through $\mathbf{R}_{6}\left(\theta_{6}\right)$ we obtain a reformulation for the relative kinematic map (57) in terms of pure rotations, only.

$$
\mathbf{D}_{H}(\boldsymbol{q})=\mathbf{R}_{6}^{-1}\left(\theta_{6}\right) \ldots \mathbf{R}_{1}^{-1}\left(\theta_{1}\right)
$$

Next we introduce the projective forward kinematic model of an articulated mechanism and prove the following proposition:

Proposition 5 The projective kinematic map of a robot manipulator is the product of projective displacements, each projective displacement being either a projective translation or a projective rotation describing a prismatic or a revolute joint. For a six degrees of freedom manipulator with revolute joints we have:

$\mathbf{H}(\boldsymbol{q})=\mathbf{H}_{1}\left(\theta_{1}\right) \mathbf{H}_{2}\left(\theta_{2}\right) \mathbf{H}_{3}\left(\theta_{3}\right) \mathbf{H}_{4}\left(\theta_{4}\right) \mathbf{H}_{5}\left(\theta_{5}\right) \mathbf{H}_{6}\left(\theta_{6}\right)$

Proof: The relative forward kinematic map describes the motion of the end-effector when the starting position is defined by the zero-reference. If this motion is observed by the two cameras, the relationship between the apparent projective displacement of the end-effector $\mathbf{H}(\boldsymbol{q})$ and the relative kinematic map is given by eq. (23) which in this particular case writes as:

$\mathbf{H}(\boldsymbol{q})=\mathbf{H}_{P E}^{-1} \mathbf{T}_{H E}^{-1} \mathbf{D}_{H}^{-1}(\boldsymbol{q}) \mathbf{T}_{H E} \mathbf{H}_{P E}=\mathbf{H}_{J}^{-1} \mathbf{D}_{H}^{-1}(\boldsymbol{q}) \mathbf{H}_{J}$

Recall that matrix $\mathbf{H}_{P E}$ stands for the projective to Euclidean upgrade and matrix $\mathbf{T}_{H E}$ describes the rigid transformation from hand (end-effector) frame to the stereo frame when the robot is in zero position, e.g., Figure 4. By expanding $\mathbf{D}_{H}^{-1}(\boldsymbol{q})$, i.e., eq. (60) we obtain:

$$
\begin{aligned}
\mathbf{H}(\boldsymbol{q}) & =\mathbf{H}_{J}^{-1}\left(\mathbf{R}_{1}\left(\theta_{1}\right) \cdots \mathbf{R}_{6}\left(\theta_{6}\right)\right) \mathbf{H}_{J} \\
& =\mathbf{H}_{J}^{-1} \mathbf{R}_{1}\left(\theta_{1}\right) \mathbf{H}_{J} \mathbf{H}_{J}^{-1} \cdots \mathbf{H}_{J} \mathbf{H}_{J}^{-1} \mathbf{R}_{6}\left(\theta_{6}\right) \mathbf{H}_{J} \\
& =\mathbf{H}_{1}\left(\theta_{1}\right) \cdots \mathbf{H}_{6}\left(\theta_{6}\right)
\end{aligned}
$$

Matrix $\mathbf{H}(\boldsymbol{q})$ describes the projective forward kinematic model of the robot-stereo configuration. Notice that each individual transformation $\mathbf{H}_{i}\left(\theta_{i}\right)$ 
has the form $\mathbf{H}_{J}^{-1} \mathbf{R}_{i}\left(\theta_{i}\right) \mathbf{H}_{J}$. Since matrix $\mathbf{R}_{i}$ describes a pure rotation $\mathbf{H}_{i}$ can be parameterized as a projective rotation $\mathbf{H}_{R}$, eq. (48). Hence, $\mathbf{H}(\boldsymbol{q})$ can be written as a product of exponentials, i.e., eq. (53) and eq. (37):

$$
\mathbf{H}(\boldsymbol{q})=\exp \left(\theta_{6} \hat{\mathbf{H}}_{6}\right) \cdots \exp \left(\theta_{1} \hat{\mathbf{H}}_{1}\right) \text {.- q.e.d. }
$$

The projective kinematic map that we just derived is valid for robots with revolute joints. Notice however that the treatment of prismatic joints is straightforward due to the algebraic properties of projective translations.

\section{The projective Jacobian matrix}

Once the direct projective kinematic map of the robot-stereo configuration has been established, it is possible to derive the relationship between jointspace velocities and image-plane velocities. The joint-to-image Jacobian matrix is a linear approximation of this relationship locally valid around a given robot configuration $\boldsymbol{q}$.

More precisely, when the robot moves, a point feature $M$ on the end-effector moves with some linear velocity. Moreover, this point is observed in both images and let $m$ and $m^{\prime}$ be its projections in the left and right image. Let's express the interaction between the joint-space velocities $\dot{\boldsymbol{q}}$ and the image-velocities $\dot{\boldsymbol{s}}$ and $\dot{\boldsymbol{s}}^{\prime}$ associated with $m$ and $m^{\prime}$ by the Jacobian $\mathbf{J}(\boldsymbol{q}, \boldsymbol{M})$ in formula:

$$
\left(\begin{array}{c}
\dot{s} \\
\dot{s}^{\prime}
\end{array}\right)=\mathbf{J}(\boldsymbol{q}, M) \dot{\boldsymbol{q}}
$$

For a six degree-of-freedom robot, $\dot{\boldsymbol{q}}$ is a 6 -vector. Since the robot is being observed in two images, the image-plane velocities $\dot{\boldsymbol{s}}$ and $\dot{\boldsymbol{s}}^{\prime}$ form a 4 -vector. Hence, the Jacobian matrix for a single featurepoint $M$ is a $4 \times 6$ matrix. Below we develop an analytic expression for $\mathbf{J}$ in $\boldsymbol{q}$ and $\boldsymbol{M}$.

As in the previous section, we consider the robot in two among its possible configurations: a general configuration which is characterized by the joint variable $\boldsymbol{q}$ and the zero-configuration for which $\boldsymbol{q}=\mathbf{0}$. Let $\boldsymbol{M}(\boldsymbol{q})$ and $\boldsymbol{M}(\mathbf{0})$ be the $3-\mathrm{D}$ projective coordinates associated with point $M$ in these two configurations. These projective coordinates can be recover by projective reconstruction (18).

$$
\begin{array}{r}
\mu \boldsymbol{m}(\boldsymbol{q})=\mathbf{P} \boldsymbol{M}(\boldsymbol{q}) \\
\mu^{\prime} \boldsymbol{m}^{\prime}(\boldsymbol{q})=\mathbf{P}^{\prime} \boldsymbol{M}(\boldsymbol{q})
\end{array}
$$

Obviously, there is a similar expression for $\boldsymbol{q}=$ 0. Due to the projective kinematic map associated with the robot, one can write:

$$
\boldsymbol{M}(\boldsymbol{q}) \simeq \mathbf{H}(\boldsymbol{q}) \boldsymbol{M}(\mathbf{0})
$$

The Matrix $\mathbf{H}(\boldsymbol{q})$ in this relation is a projective displacement, therefore it is a normalized homography. Without loss of generality, one can associate a fix scale $\rho$, and hence obtains a fixed vector $\boldsymbol{M ( 0 )}$ the following strict equality (see eq. (28)):

$$
M_{\rho}(\boldsymbol{q})=\mathbf{H}(\boldsymbol{q}) \boldsymbol{M}_{\rho}(\mathbf{0})
$$

Moreover, the projective equalities of equations (65) and (66) become standard equalities:

$$
\begin{array}{r}
\boldsymbol{m}_{\rho}(\boldsymbol{q})=\mathbf{P} \boldsymbol{M}_{\rho}(\boldsymbol{q}) \\
\boldsymbol{m}_{\rho}^{\prime}(\boldsymbol{q})=\mathbf{P}^{\prime} \boldsymbol{M}_{\rho}(\boldsymbol{q})
\end{array}
$$

The velocity $\dot{\boldsymbol{M}}_{\rho}(t)$ of $\boldsymbol{M}_{\rho}(t)$ was introduced in section 4.4 . This velocity is related to the robotjoint velocities and to the image-plane velocities in the following way.

First we consider the relationship between the image-plane velocities and $\dot{\boldsymbol{M}}_{\rho}(t)$. Notice that the projection matrices $\mathbf{P}$ and $\mathbf{P}^{\prime}$ in eqs. (68) and (69) do not vary over time because of the rigidity of the stereo rig. We can therefore take the time derivative and obtain:

$$
\begin{aligned}
& \dot{\boldsymbol{m}}_{\rho}(\boldsymbol{q})=\mathbf{P} \dot{\boldsymbol{M}}_{\rho}(\boldsymbol{q}) \\
& \dot{\boldsymbol{m}}_{\rho}^{\prime}(\boldsymbol{q})=\mathbf{P}^{\prime} \dot{\boldsymbol{M}}_{\rho}(\boldsymbol{q})
\end{aligned}
$$

Let the coordinates of the 3-vector $\boldsymbol{m}_{\rho}(\boldsymbol{q})$ be $\left(m_{1}, m_{2}, m_{3}\right)$. These are the homogeneous coordinates of an image point. The corresponding pixel coordinates of this point are obtained by dividing the first and second coordinates with the third coordinate, $\boldsymbol{s}=\left(m_{1} / m_{3}, m_{2} / m_{3}\right)$. We have a similar expression for $\boldsymbol{s}^{\prime}$. By differentiating these two vec- 
tors we obtain:

$$
\left(\begin{array}{c}
\dot{\boldsymbol{s}} \\
\dot{\boldsymbol{s}}^{\prime}
\end{array}\right)=\left[\begin{array}{cccccc}
\frac{1}{m_{3}} & 0 & -\frac{m_{1}}{m_{3}^{2}} & 0 & 0 & 0 \\
0 & \frac{1}{m_{3}} & -\frac{m_{2}}{m_{3}^{2}} & 0 & 0 & 0 \\
0 & 0 & 0 & \frac{1}{m^{\prime} 3} & 0 & -\frac{m_{1}^{\prime}}{m^{\prime 2}} \\
0 & 0 & 0 & 0 & \frac{1}{m_{3}^{\prime}} & -\frac{m_{2}^{\prime}}{m_{3}^{\prime 2}}
\end{array}\right]\left[\begin{array}{c}
\mathbf{P} \\
\mathbf{P}^{\prime}
\end{array}\right] \dot{\boldsymbol{M}}_{\rho}(\boldsymbol{q})
$$

Second we consider the relationship between $\dot{\boldsymbol{M}}_{\rho}(t)$ and the robot-joint velocity vector $\dot{\boldsymbol{q}}$. We have:

$$
\dot{\boldsymbol{M}}_{\rho}(\boldsymbol{q})=\frac{d \boldsymbol{M}_{\rho}(\boldsymbol{q})}{d t}=\frac{d \boldsymbol{M}_{\rho}(\boldsymbol{q})}{d \boldsymbol{q}} \frac{d \boldsymbol{q}}{d t}=\frac{d \boldsymbol{M}_{\rho}(\boldsymbol{q})}{d \boldsymbol{q}} \dot{\boldsymbol{q}}
$$

The derivatives of $\boldsymbol{M}_{\rho}$ with respect to the joint variables write as a $4 \times 6$ matrix:

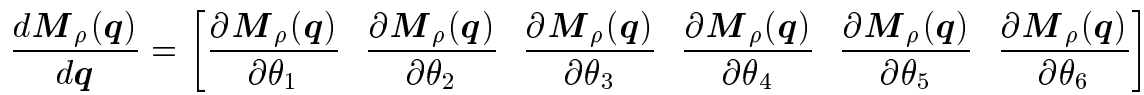

Let us further consider the partial derivative of $\boldsymbol{M}_{\rho}$ with respect to $\theta_{i}$ :

$$
\begin{aligned}
\frac{\partial \boldsymbol{M}_{\rho}(\boldsymbol{q})}{\partial \theta_{i}} & =\frac{\partial}{\partial \theta_{i}} \mathbf{H}(\boldsymbol{q}) \boldsymbol{M}_{\rho}(\mathbf{0}) \\
& =\mathbf{H}_{1}\left(\theta_{1}\right) \ldots \frac{d \mathbf{H}_{i}\left(\theta_{i}\right)}{\theta_{i}} \ldots \mathbf{H}_{6}\left(\theta_{6}\right) \boldsymbol{M}_{\rho}(\mathbf{0}) \\
& =\mathbf{H}_{1}\left(\theta_{1}\right) \ldots \hat{\mathbf{H}}_{i} \mathbf{H}_{i}\left(\theta_{i}\right) \ldots \mathbf{H}_{6}\left(\theta_{6}\right) \boldsymbol{M}_{k}(\mathbf{0} \mathbf{0})
\end{aligned}
$$

Indeed, the normalized homography $\mathbf{H}(\boldsymbol{q})$ describing the direct kinematic model of the robot is a product of 6 projective rotations. Each one of these matrices has Lie-group structure and, as shown in section 6.2 , they can be parameterized by the angle of rotation $\theta_{i}$ and the tangent operator $\hat{\mathbf{H}}_{i}$.

Finally, the expression of the Jacobian matrix mapping joint angular velocities to image-plane point velocities is:

$\mathbf{J}(\boldsymbol{q}, \boldsymbol{M})=\left[\begin{array}{cccccc}\frac{1}{m_{3}} & 0 & -\frac{m_{1}}{m_{3}^{2}} & 0 & 0 & 0 \\ 0 & \frac{1}{m_{3}} & -\frac{m_{2}}{m_{3}^{2}} & 0 & 0 & 0 \\ 0 & 0 & 0 & \frac{1}{m^{\prime} 3} & 0 & -\frac{m_{1}^{\prime}}{m^{\prime 2}} \\ 0 & 0 & 0 & 0 & \frac{1}{m_{3}^{\prime}} & -\frac{m_{2}^{3}}{m_{3}^{\prime 2}}\end{array}\right]$

In this section we devise algebraic and numerical methods for recovering the projective kinematic map from trial motions of a robot. More precisely, for each joint we seek to estimate the parameters underlying the tangent operator $\hat{\mathbf{H}}_{i}$ corresponding to each projective rotation $\mathbf{H}_{i}\left(\theta_{i}\right)$. A [pucticadmander to acquire the trial data is to mBre] the joot joint by joint (Fig. 6), and to capture the respective projective motion of endeffector point-features (Fig. 7) by means of the 

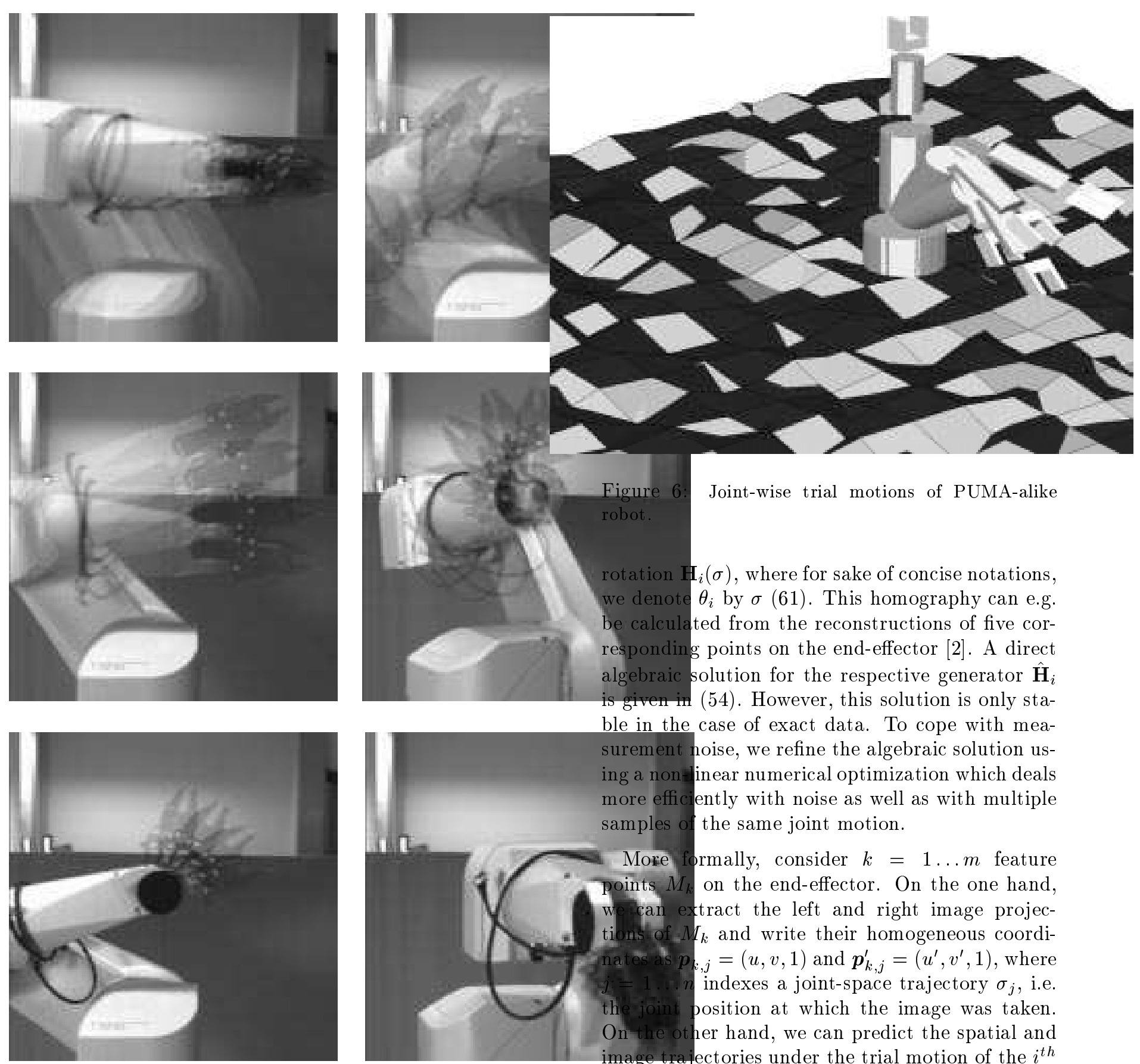

Figure 6

robot

rotation $\mathbf{H}_{i}(\sigma)$, where for sake of concise notations, we denote $\theta_{i}$ by $\sigma(61)$. This homography can e.g. be calculated from the reconstructions of five corresponding points on the end-effector [2]. A direct algebraic solution for the respective generator $\hat{\mathbf{H}}_{i}$ is given in (54). However, this solution is only stable in the case of exact data. To cope with measurement noise, we refine the algebraic solution using a non-linear numerical optimization which deals more efficiently with noise as well as with multiple samples of the same joint motion.

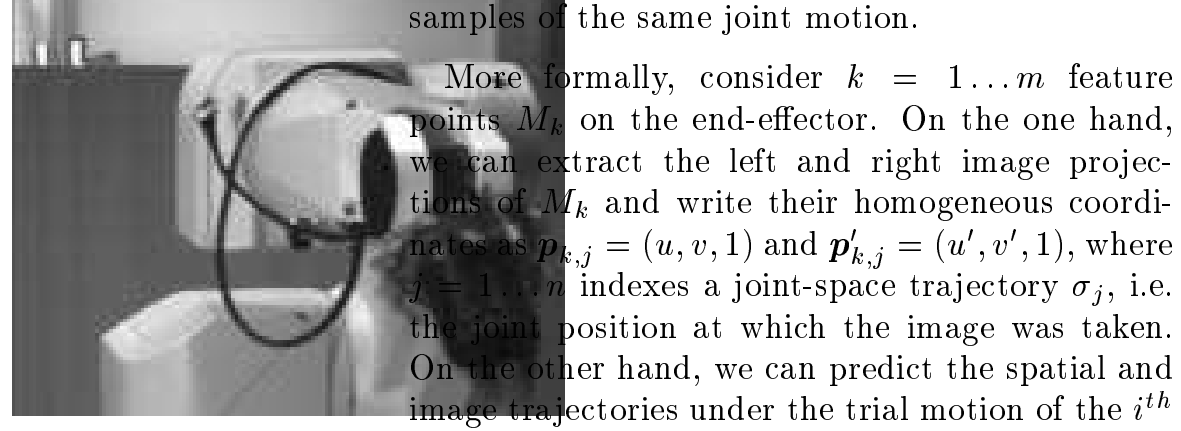

Figure 5: Images of joint-wise trial motions of Stäubli RX90 robot at INRIA Rhône-Alpes.

joint using eqs. (8), (68), (69):

$$
\boldsymbol{m}_{k, j}=\mathbf{P} \underbrace{\exp \left(\sigma_{j} \hat{\mathbf{H}}_{i}\right)}_{\mathbf{H}_{i}\left(\sigma_{j}\right)} \boldsymbol{M}_{k}, \quad \boldsymbol{m}_{k, j}^{\prime}=\mathbf{P}^{\prime} \underbrace{\exp \left(\sigma_{j} \hat{\mathbf{H}}_{i}\right)}_{\mathbf{H}_{i}\left(\sigma_{j}\right)} \boldsymbol{M}_{k} \text {. }
$$

In order to estimate the generator matrix, an objective function $f$ is formulated in $\hat{\mathbf{H}}_{i}$. It calculates 


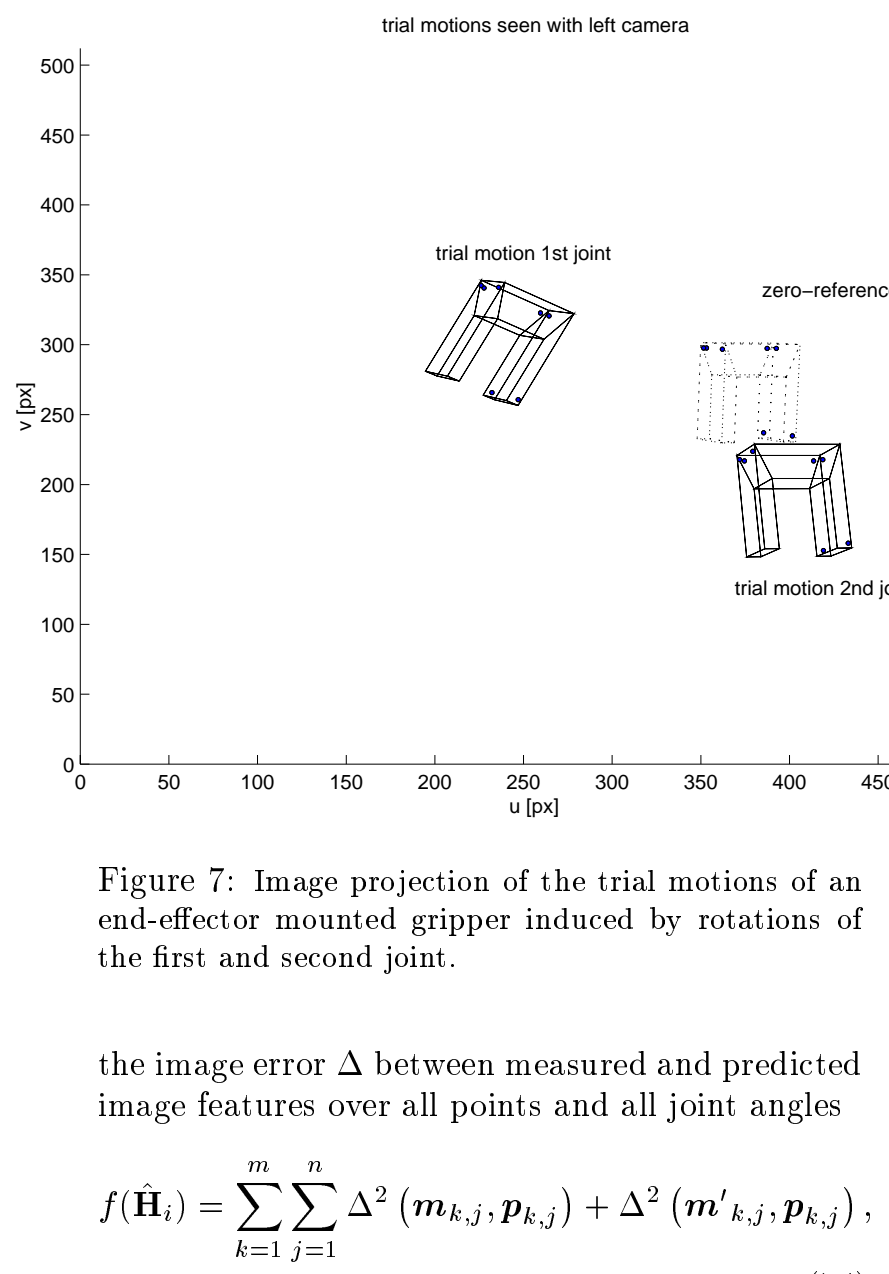

where e.g. $\Delta(\boldsymbol{m}, \boldsymbol{p})=\left(\left(\frac{m_{1}}{m_{3}}-u\right)^{2}+\left(\frac{m_{2}}{m_{3}}-v\right)^{2}\right)^{1 / 2}$ is the Euclidean image error. In summary, given joint angle measurements and the image data acquired from the trial motions, non-linear leastsquares are used to minimize $f$ over the entries in $\hat{\mathbf{H}}_{i}$. This procedure is repeated for each robot joint.

\subsection{Minimal parameterization}

Now, we introduce a minimal parameterization of a generator $\hat{\mathbf{H}}$ based on the 16 coefficients in the vectors $\boldsymbol{k}_{1}, \boldsymbol{k}_{2}, \boldsymbol{h}_{1}^{\top}, \boldsymbol{h}_{2}^{\top}$ in (49):

$\hat{\mathbf{H}}(\boldsymbol{x})=\left[\begin{array}{ll}k_{11} & k_{12} \\ k_{21} & k_{22} \\ k_{13} & k_{23} \\ k_{14} & k_{24}\end{array}\right] \mathbf{C}_{a b}^{-1}\left[\begin{array}{cc}0 & -1 \\ 1 & 0\end{array}\right] \mathbf{C}_{a b}\left[\begin{array}{llll}h_{11} & h_{12} & h_{13} & h_{14} \\ h_{21} & h_{22} & h_{23} & h_{24}\end{array}\right]_{\mathrm{wh}}$,

(75) $\boldsymbol{e}_{-i}^{\top}, \boldsymbol{e}_{i}^{\top}$ to the eigenvalues $-i$ and $i$ are recombined where $\mathbf{C}_{a b}=\left[\begin{array}{cc}a-b \\ b & a\end{array}\right]$ follows from the commutator $\mathbf{C}_{R}$ (12). Four of these parameters are constrained by (32) and further two parameters are absorbed through the gauge-freedom $\mathbf{C}_{a b}$ in $a$ and $b$. These two are eliminated by fixing two parameters, e.g. $h_{11}=1, h_{21}=0$. In practice, we impose this $\left[\begin{array}{l}\boldsymbol{h}_{1}^{\top} \\ \boldsymbol{h}_{2}^{\top}\end{array}\right]=\mathbf{Q} \cdot\left[\begin{array}{llll}1 & h_{12}^{\prime} & h_{13}^{\prime} & h_{14}^{\prime} \\ 0 & h_{22}^{\prime} & h_{23}^{\prime} & h_{24}^{\prime}\end{array}\right]=\mathbf{Q}\left[\begin{array}{l}\boldsymbol{h}_{1}^{\prime \top} \\ \boldsymbol{h}_{2}^{\prime \top}\end{array}\right]$.

As soon as we identify $\mathbf{C}_{a b}=\mathbf{Q}^{-1}$ and update $\left[\begin{array}{ll}\boldsymbol{k}_{1}^{\prime} & \boldsymbol{k}_{2}^{\prime}\end{array}\right]=\left[\begin{array}{ll}\boldsymbol{k}_{1} & \boldsymbol{k}_{2}\end{array}\right] \mathbf{Q}$, the generator matrix $\hat{\boldsymbol{H}}$ is $\boldsymbol{x}=\left(h_{12}^{\prime}, h_{13}^{\prime}, h_{14}^{\prime}, h_{22}^{\prime}, h_{23}^{\prime}, h_{24}^{\prime}, k_{13}^{\prime}, k_{14}^{\prime}, k_{23}^{\prime}, k_{24}^{\prime}\right)^{T}$

$$
\underset{\frac{1}{\mathbf{H}}(\boldsymbol{x})}{\mathbf{5 0 0}}=\left[\begin{array}{lll}
k_{11}^{\prime} & k_{12}^{\prime} \\
k_{12}^{\prime} & k_{22}^{\prime} \\
k_{13}^{\prime} & k_{23}^{\prime} \\
k_{14}^{\prime} & k_{24}^{\prime}
\end{array}\right]\left[\begin{array}{cc}
0 & -1 \\
1 & 0
\end{array}\right]\left[\begin{array}{cccc}
1 & h_{12}^{\prime} & h_{13}^{\prime} & h_{14}^{\prime} \\
0 & h_{22}^{\prime} & h_{23}^{\prime} & h_{24}^{\prime}
\end{array}\right],
$$

since the bilinear constraints (32) in the simplified form

$$
\left[\begin{array}{llll}
1 & h_{12}^{\prime} & h_{13}^{\prime} & h_{14}^{\prime} \\
0 & h_{22}^{\prime} & h_{23}^{\prime} & h_{24}^{\prime}
\end{array}\right]\left[\begin{array}{ll}
\boldsymbol{k}_{1}^{\prime} & \boldsymbol{k}_{2}^{\prime}
\end{array}\right]=\left[\begin{array}{ll}
1 & 0 \\
0 & 1
\end{array}\right],
$$

are easily solved for $k_{11}^{\prime}, k_{12}^{\prime}, k_{21}^{\prime}, k_{22}^{\prime}$. Finally, the objective function becomes $f(\boldsymbol{x})=f\left(\hat{\mathbf{H}}_{i}(\boldsymbol{x})\right)$ and is minimized over $\boldsymbol{x}$. However, during the minimization $h_{11}$ may not vanish, otherwise the $Q R$ step has to be applied repeatedly.

\subsection{Initialization}

The initialization of $\boldsymbol{x}$ from a given projective rotation $\mathbf{H}_{i}$ remains a crucial issue. It is straightforward, that any Jordan decomposition (39) yields an initial guess for $\boldsymbol{x}$, but its numerical calculation is very unstable. In contrast, the tangent operator $\hat{\mathbf{H}}_{R}$ calculated using first (54) and imposing then rank 2 by SVD usually gives better results. But still, $\boldsymbol{x}$ has to be recovered from $\hat{\mathbf{H}}_{R}$ by eigendecomposition

$$
\hat{\mathbf{H}}_{R}=\left[\boldsymbol{f}_{-_{i}} \boldsymbol{f}_{i}\right]\left[\begin{array}{cc}
-i & 0 \\
0 & i
\end{array}\right]\left[\begin{array}{c}
\boldsymbol{e}_{-i}^{\top} \\
\boldsymbol{e}_{i}^{\top}
\end{array}\right],
$$
omixpressed as a function of the 10 parameter vector 
using the matrix $\Upsilon=\left[\begin{array}{cc}1 & -i \\ 1 & i\end{array}\right]$ in order to obtain the

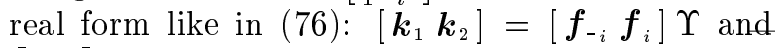
$\left[\begin{array}{l}\boldsymbol{h}_{1}^{\top} \\ \boldsymbol{h}_{2}^{\top}\end{array}\right]=\Upsilon^{-1}\left[\begin{array}{l}\boldsymbol{e}_{-i}^{\top} \\ \boldsymbol{e}_{i}^{\top}\end{array}\right]$. Now, the parameters follow at a glance.

\section{Non-metric Visual Servo- ing}

In this section we validate the above introduced projective kinematic model of a six-axes manipulator. For this purpose, we formulate and simulate an image-based visual servoing based on the Jacobian developed in section 8 . We call our formulation "non-metric" for three reasons: First, generally speaking, the geometry of the entire system is modeled with respect to a projective frame of five rigid points associated with the stereo rig. All Cartesian frames disappear. Second, no a-priori knowledge about the geometry of the system is required. The epipolar geometry, the structure of the gripper, and the projective kinematic map can be acquired from corresponding data of matched image features and joint angle measurements, without requiring neither a-priori knowledge, nor manual interventions. Third, the actual control law no longer servos the robot's Cartesian velocity, but servos the manipulator's joint-velocities. Most important is to see that the Jacobian is an analytic expression in $\boldsymbol{q}$, which ensures its soundness over the robot's entire configuration space. In contrast to existing systems, it is neither an on-line estimated linear model [18], nor an a-priori given approximation around the target [5], [7].

The system is illustrated in Figure 8 and consists of:

1. A six-axes robotic manipulator with a PUMAalike geometric structure.

The three links of the arm are of lengths $l_{1}=$ $36 \mathrm{~cm}, l_{2}=48 \mathrm{~cm}, l_{3}=40 \mathrm{~cm}$, respectively.

2. A parallel-jaw gripper on the end-effector that is marked with point features.

The corners of the gripper constitute the features since they are easy to extract and posses a wide angle of visibility. The length of the gripper is about $9 \mathrm{~cm}$. 
to both, $u$ and $v$ image-coordinates. A $\mathrm{n}$ imum of 5 point features is required for homographies $\mathbf{H}_{R}$ to be estimated, altho we use 20 points to achieve good precisior the presence of noise.

4. Recover the projective kinematic map of robot. (see section 9 )

For the minimization we took only two ste images, namely those taken at maximal an lar deflection. These are in detail $30^{\circ}, 20^{\circ}$, ؛ $40^{\circ}, 60^{\circ}, 60^{\circ}$ for the joints 1 to 6 .

5. Move the robot in a target position, tak stereo image, and extract target image featt $s_{k}^{*}, s_{k}^{*}$.

This also could have been done off-line, us a method for target transfer described in

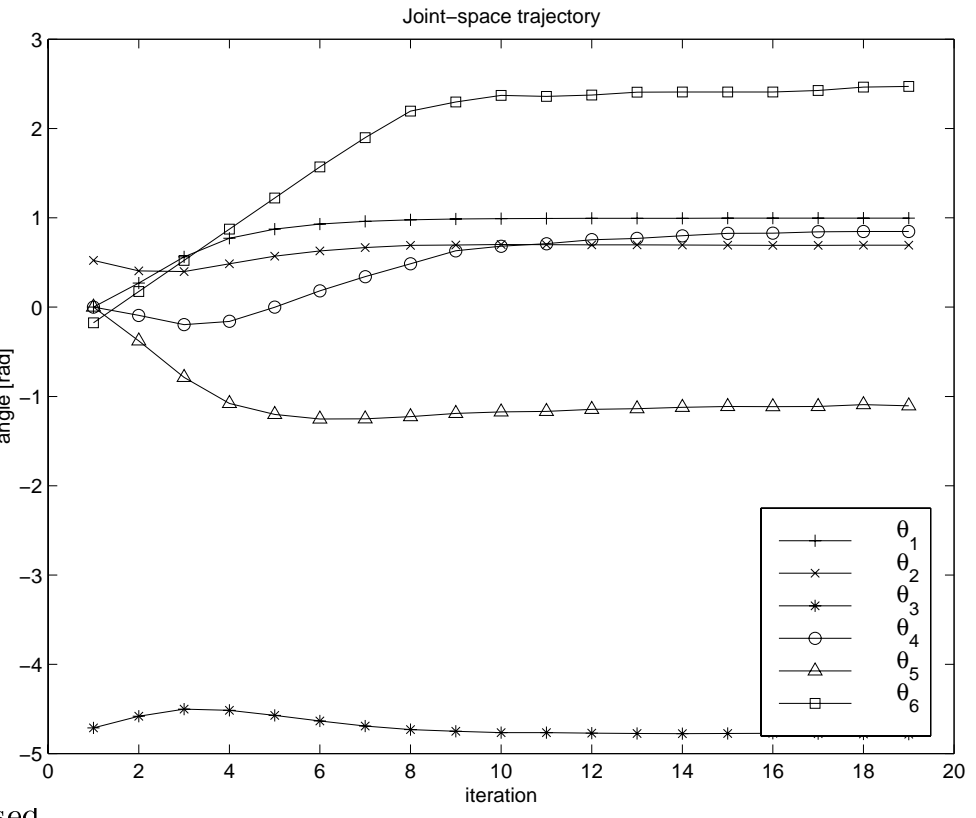
that allows different stereo systems to be used for the acquisition of the target and the servoing. In the simulations the target consists of three point features on the visible face of the gripper (Fig. 10).

6. Move the robot to a starting postion, extract and match current image features $\boldsymbol{s}_{k}, \boldsymbol{s}^{\prime}{ }_{k}$ with the target features $s_{k}^{*}, \boldsymbol{s}_{k}^{\prime *}$, thus calculate the stereo error-vector for each feature

$$
\boldsymbol{S}_{k}=\left(\begin{array}{c}
\boldsymbol{s}_{k}^{*}-\boldsymbol{s}_{k} \\
\boldsymbol{s}_{k}^{\prime *}-\boldsymbol{s}_{k}^{\prime}
\end{array}\right)
$$

A stereo pair of linear image-errors in first instance implies a linear spatial velocity of each feature point. So, aligning the robot control with the reversed error-vector generates a rigid motion such that it best fits in the least-squares sense with the three linear pointvelocities. This in particular implies that trajectories depend heavily on the configuration of the feature points and that control might converge to a locally minimal image-error, but not necessarily attain the target position. A well adapted approach to trajectory generation using uncalibrated cameras that overcomes these limitations is proposed in [25].

7. Determine joint velocities by applying the pseudo-inverse of the Jacobian to the error-

Figure 9: Joint-space trajectory of non-metric visual servoing.

vector (section 8$)$.

$$
\dot{\boldsymbol{q}}=-\left[\begin{array}{l}
\mathbf{J}\left(\boldsymbol{q}, \boldsymbol{M}_{1}\right) \\
\mathbf{J}\left(\boldsymbol{q}, \boldsymbol{M}_{2}\right) \\
\mathbf{J}\left(\boldsymbol{q}, \boldsymbol{M}_{3}\right)
\end{array}\right]^{+}\left(\begin{array}{c}
\boldsymbol{S}_{1} \\
\boldsymbol{S}_{2} \\
\boldsymbol{S}_{3}
\end{array}\right)
$$

This is done in analogy with previous work on visual servoing using stereo vision [7] or visual servoing of joint velocites [18]. We would like to stress that, in contrast to these approaches, the Jacobian employed in our approach (72) is at each instant in time a sound first order model of the interaction matrix between jointand image-velocities.

8. Run closed-loop visual servoing until the image error vanishes, i.e. until joint velocites approach zero and hence the robot stops.

The control law is designed to ensure exponential convergence in the image-error [5]. Figure 11 shows the progression of the error in $u$ - and $v$ - coordinates during servoing. After a phase of saturated joint velocites till iteration 3 , the image error decreases exponentially and the control converges after 15 iterations. The respective image trajectories can be found 
[5] B. Espiau, F. Chaumette, and P. Rives. A new approach to visual servoing in robotics. IEEE Transactions on Robotics and Automation, 8(3):313-326, June 1992.

[6] O. D. Faugeras. Three Dimensional Computer Vision: A Geometric Viewpoint. MIT Press, Boston, 1993.

[7] G. D. Hager. A modular system for robust positioning using feedback from stereo vision. IEEE Transactions on Robotics and Automation, 13(4):582-595, August 1997.

[8] R. I. Hartley. Euclidean reconstruction from uncalibrated views. In Mundy Zisserman Forsyth, editor, Applications of Invariance in Computer Vision, pages 237-256. Springer Verlag, Berlin Heidelberg, 1994.

[9] R. I. Hartley. Projective reconstruction and invariants from multiple images. IEEE Transactions on Pattern Analysis and Machine Intelligence, 16(10):1036-1041, October 1994.

[10] R. I. Hartley. Self-calibration of stationary cameras. International Journal of Computer Vision, 22(1):5-23, February/March 1997.

[11] N. Hollinghurst and R. Cipolla. Uncalibrated stereo hand-eye coordination. Image and $\mathrm{Vi}$ sion Computing, 12(3):187-192, March 1994.

[12] R. Horaud and G. Csurka. Self-calibration and Euclidean reconstruction using motions of a stereo rig. In Proceedings Sixth International Conference on Computer Vision, pages 96-103, Bombay, India, January 1998. IEEE Computer Society Press, Los Alamitos, Ca.

[13] R. Horaud, G. Csurka, and D. Demirdjian. Stereo calibration from rigid motions. Technical Report RR-3467, INRIA, June 1998. Submitted to IEEE Trans. on Pattern Analysis and Machine Intelligence.

[14] R. Horaud and F. Dornaika. Hand-eye calibration. International Journal of Robotics Research, 14(3):195-210, June 1995.

[15] R. Horaud, F. Dornaika, and B. Espiau. Visually guided object grasping. IEEE Transactions on Robotics and Automation, 14(4):525532, August 1998.
[16] R. A. Horn and C. A. Johnson. Matrix Analysis. Cambridge University Press, Cambridge, 1994.

[17] S. Hutchinson, G. D. Hager, and P. I. Corke. A tutorial on visual servo control. IEEE Transactions on Robotics and Automation, 12(5):651-670, October 1996.

[18] M. Jagersand and R. Nelson. Aquiring visualmotor models for precision manipulation with robot hands. In Buxton-Cipolla, editor, Computer Vision - ECCV'96, Proceedings Fourth European Conference on Computer Vision, Cambridge, England, volume II, pages 603612. Springer Verlag, April 1996.

[19] K. Kinoshita. Visual servoing with uncalibrated cameras. In The Fourth International Conference on Control, Automation, Robotics, and Vision, pages 1705-1709, Singapore, December 1996.

[20] Q-T. Luong and O. D. Faugeras. The fundamental matrix: Theory, algorithms, and stability analysis. International Journal of Computer Vision, 17(1):43-75, 1996.

[21] Q-T. Luong and O. D. Faugeras. Selfcalibration of a moving camera from point correspondences and fundamental matrices. International Journal of Computer Vision, 22(3):261-289, 1997.

[22] B.W. Mooring, Z.S. Roth, and M.R. Driels. Fundamentals of Manipulator Calibration. John Wiley \& Sons, New York, 1991.

[23] M. Pollefeys, R. Koch, and L. Van Gool. Selfcalibration and metric reconstruction in spite of varying and unknown internal camera parameters. In Proceedings Sixth International Conference on Computer Vision, pages 90-95, Bombay, India, January 1998. IEEE Computer Society Press, Los Alamitos, Ca.

[24] A. Ruf, G. Csurka, and R. Horaud. Projective translations and affine stereo calibration. In Proceedings IEEE Conference on Computer Vision and Pattern Recognition, pages 475481, Santa Barbara, CA, June 1998. IEEE Computer Society Press, Los Alamitos, Ca. 
[25] Andreas Ruf. Visual trajectories from uncalibrated images. In Workshop on New Trends in Image-Based Robot Servoing, 1997.

[26] C. Samson, M. Le Borgne, and B. Espiau. Robot Control, the Task Function Approach. Oxford Science Publications, Oxford, 1991.

[27] Z. Zhang, R. Deriche, O. D. Faugeras, and QT. Luong. A robust technique for matching two uncalibrated images through the recovery of the unknown epipolar geometry. Artificial Intelligence, 78(1-2):87-119, October 1995.

[28] H. Zhuang, K. Wang, and Z. S. Roth. Simultaneous calibration of a robot and a handmounted camera. IEEE Transactions on Robotics and Automation, 11(5):649-660, October 1995.

[29] A. Zisserman, P. A. Beardsley, and I. D. Reid. Metric calibration of a stereo rig. In Proc. IEEE Workshop on Representation of Visual Scenes, pages 93-100, Cambridge, Mass., June 1995. 